This is the final peer-reviewed accepted manuscript of:

Formentin, S.M. \& Zanuttigh, B., 2018. A new method to estimate the overtopping and overflow discharge at over-washed and breached dikes. Coastal engineering, 140, pp.240-256.

The final published version is available online at:

https://doi.org/10.1016/j.coastaleng.2018.08.002

Rights / License:

The terms and conditions for the reuse of this version of the manuscript are specified in the publishing policy. For all terms of use and more information see the publisher's website.

This item was downloaded from IRIS Università di Bologna (https://cris.unibo.it/)

When citing, please refer to the published version. 


\title{
A NEW METHOD TO ESTIMATE THE OVERTOPPING AND OVERFLOW DISCHARGE AT OVER-WASHED AND BREACHED DIKES
}

\author{
Sara Mizar Formentin ${ }^{1}$, Barbara Zanuttigh ${ }^{1,2}$
}

(1) Department of Civil, Chemical, Environmental and Materials Engineering, University of Bologna, Viale del Risorgimento 2, Bologna 40136, Italy. saramizar.formentin2@unibo.it, barbara.zanuttigh@unibo.it

(2) Delft University of Technology, Stevinweg 1, Delft, The Netherlands, B.Zanuttigh@tudelft.nl

\begin{abstract}
Most of the experience collected on wave overtopping is concentrated on emerged or zerofreeboard structures, and only few experiments are available on over-washed dikes. The experience on submerged crest conditions is principally dedicated to rubble mound structures and limited to the wave reflection and transmission processes. The existing tools for the prediction of the average wave overtopping discharge $(q)$ at dikes are therefore targeted to represent overtopped structures, leading to cautious estimates at over-washed dikes and unrealistic overestimations in case of catastrophic scenarios such as breached dikes. This paper proposes a new conceptual and practical method for the prediction of $q$ that is valid for both overwashed and fully breached dikes. It consists of three physically-based formulae whose coefficients are fitted on a new numerical database of smooth structures under variable wave attack. The predictions are compared with the new numerical results and the data and the tools available from the literature, i.e. experimental data, formulae and neural network. The new method gives a more realistic representation of the overtopping and overflow processes, reducing significantly the overestimation in case of breaching and being at least as accurate as the existing tools in case of over-washing.
\end{abstract}

Keywords: wave overtopping discharge; overflow; breaching; dike; conceptual method; climate change. 


\section{Highlights}

An innovative method is proposed for the estimation of wave overtopping and overflow discharge at dikes

The method can be applied to over-washed and breached dikes

The method is based on a coherent theoretical and physically-based framework

The method consists of three equations for a practical application 


\section{Introduction}

Earthen levees and dikes are used throughout the world to protect populations and infrastructures from periodic floods and high water due to storm surges. Ideally, the height of these structures should be adequate to prevent flood or storm surge overflow and/or wave overtopping for any storm scenario. However, economic constraints and environmental impacts usually impose more practical designs with lower crown elevations, accepting the associated risk that some wave/surge overtopping will occur during extreme events. The increase of the storm frequency and intensity induced by climate change, combined with the uncertainties related to extreme events and to climate variability, pose serious challenges to the long term design. It is likely that in the next future many dikes will operate for longer times at lower crest freeboards, i.e. close to mean sea level or even over-washed. Moreover, catastrophic events, such as the Hurricane Katrina (Seed et al., 2008), may cause the partial or complete breaching of the levees.

Therefore, for design purposes, accurate estimates of the average overtopping rate for a set of climate conditions are needed to assess accurately the wave energy reduction and the safety level of the inland area and to minimize construction costs.

For emerged structures, the database collected within the FP5 CLASH project (Van der Meer et al., 2009 (b)) and recently updated and extended by Zanuttigh et al. (2016), provides a wide experimental information on the wave overtopping discharge $(q)$. Such database includes nearly 18,000 laboratories measurements of $q$ and is available upon registration at www.unibo.it/overtopping-neuralnetwork. Based on these data, EurOtop (2016) proposes the full range of formulae for permeable and impermeable emerged structures with gentle, steep and vertical slope. The neural networks for wave overtopping (Van Gent et al., 2007; Formentin et al., 2017) can deal with very complex geometries but cannot represent with confidence neither over-washed nor submerged conditions.

For over-washed dikes, the information available so far is limited to the set of tests performed by Hughes \& Nadal (2009) and Hughes et al. (2012). The authors provided a formula for estimating $q$ that is based on the weir-like approximation proposed by EurOtop (2007) but it has been fitted on their data. The same formula adopted by EurOtop (2007) is recalled by EurOtop (2016).

As for low-crest or submerged conditions, research was carried out during the FP5 project DELOS (Kramer et al., 2005), limitedly in most cases to wave reflection and transmission (Van der Meer et al., 2005). This experimental analysis prompted the calibration of numerical models (Johnson et al., 2005) and the development of conceptual approaches (Zanuttigh et al., 2008) but did not lead to the release of a formula for estimating $q$ for any submergence.

The overtopping process depends also on the structure landward conditions. The presence of water behind the breached dikes ("wet" landward conditions, from now on; see the scheme of Figure 1) determines a significant reduction of the hydraulic head between the off-shore and the in-shore sections of the structure crest and may generate piling-up and therefore return flow over 
the crest. The result is that the value of $q$ is significantly lower for the breached structure than for the same (i.e. same geometry) over-washed structure (i.e. characterized by "dry" landward conditions, from now on), exposed to the same wave conditions. The application of the weir-like formulae to structures in wet landward conditions may lead to overestimations of $q$ and in turns to inaccurate predictions of the flooding consequent to the complete failure of the dikes.
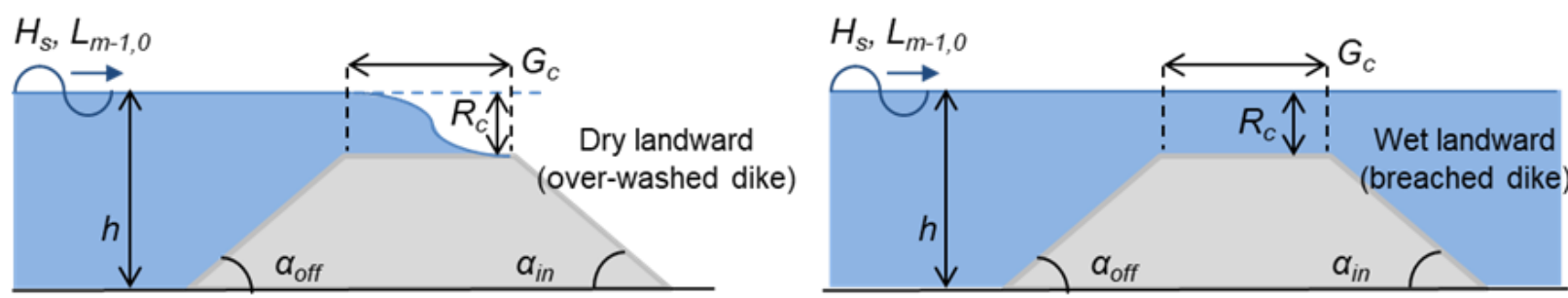

Figure 1 - Schematic layout of the dike cross-sections with reference to the adopted symbol and display of the "dry" and the "wet" landward conditions.

The goal of this paper is to provide engineers and designers with a complete and innovative method for the estimation of the overtopping and overflow discharges behind over-washed and breached dikes. To this purpose, a systematic study was performed by means of numerical modelling and a novel method was developed based on the new numerical database and verified against existing experimental data.

The paper structure is as follows. It starts (Section 2) with a short description of the model and presents the new numerical database, identifying the key parameters for the set-up of the numerical simulations. This database was set up by running the RANS-VOF code (IH-2VOF) developed by the University of Cantabria (Losada et al., 2005; Lara et al., 2008) in presence of smooth impermeable over-washed and breached structures. Above all, 94 tests were performed, by varying the structures off-shore slope, the crest freeboard and the wave attack. The validation of the numerical model is given by reporting the results of the simulation of 5 experimental tests of wave overtopping on over-washed dikes in dry landward conditions and 5 experimental tests of wave overtopping on submerged dikes in wet landward conditions. The verification of the accuracy of the numerical results is provided by comparing the values of the wave reflection and wave transmission coefficients $\left(K_{r}\right.$ and $\left.K_{t}\right)$ derived from the 94 numerical simulations with similar laboratory tests available from the literature and with the predictions prompted by the ANN tool developed by the authors (Zanuttigh et al., 2016; Formentin et al., 2017). Section 3 proposes an overview of the current methods available for the prediction of $q$, i.e. the formulae by EurOtop (2016) and by Hughes \& Nadal (2009). The Section points out and discusses the limits of applicability of these methods, which are targeted to represent emerged or over-washed structures only. The new method, proposed in Section 4, has been specifically conceived to give a more appropriate representation of the overflow process that occurs when the hydraulic head between the off-shore and the in-shore sections of the structure is reduced by the presence of the water behind the structure itself. The illustration of the new method includes the presentation of the results and the discussion of its performance. The validity of the method is then checked 
against laboratory data on over-washed dikes in dry landward conditions and on rubble mound low-crested structures (LCS) in wet landward conditions (Section 5). An example application of the new method, presenting and detailing the conceptual steps required for the use of the method itself, is proposed in Section 6. Finally, Section 7 draws some conclusions on the relevance of these results for design purpose. The full list of symbols adopted in the paper is provided in Section 8.

\section{Setup of the numerical model}

This section aims at providing the reader with synthetic information about the numerical model adopted (Sub-section 2.1) and the tested hydraulic and geometrical conditions (Sub-section 2.2). The capacity and adequacy of the model to reproduce the overtopping processes in case of both over-washed and breached dikes is validated against experimental data ( Sub-section 2.3). A preliminary verification of the reliability and consistency of the results obtained from the new numerical simulations is illustrated in Sub-section 2.4 .

\subsection{Short description of the model}

The model used in this work is the IH-2VOF (Lara et al., 2011) that is a modified and improved version of COBRAS-UC (Losada et al., 2005), developed on the basis of COBRAS (Liu \& Lin, 1997) and RIPPLE (Kothe et al., 1991) codes. The model has undergone a continuous development and an extensive validation procedure, carried out for low-crested structures (Garcia et al., 2004), wave breaking on permeable slopes (Lara et al., 2006), surf zone hydrodynamics on natural beaches (Torres-Freyermuth et al., 2007) and wave overtopping (Losada et al., 2005; Lara et al., 2008).

This kind of models solves the 2DV Reynolds Average Navier-Stokes (RANS) equations, based on the decomposition of the instantaneous velocity and pressure fields, into average and turbulent components. The $k-\varepsilon$ equations (Rodi, 1980; Lin and Liu, 1998) are used for modeling the turbulent kinetic energy $(k)$ and the turbulent dissipation rate $(\varepsilon)$. The influence of turbulence fluctuations on the mean flow field is represented by the Reynolds stresses. The governing equations for $k-\varepsilon$ are derived from the Navier-Stokes equations, and higher order correlations of turbulence fluctuations in $K$ and $\varepsilon$ equations are replaced by closure conditions. A non-linear algebraic Reynolds stress model is used to relate the Reynolds stress tensor and the strain rate of mean flow. The free surface movement is tracked by the Volume of Fluid (VOF) method. The flow inside the porous media is solved through the resolution of the Volume-Averaged Reynolds Averaged Navier-Stokes equations. These equations are derived by integrating the RANS equations over a control volume and the interfacial forces between the fluid and solids have been modeled according to the extended Forchheimer relationship, in which both linear and nonlinear 
drag forces are included in the equations. For the complete mathematical formulation see Liu et al. (1999) and Hsu et al. (2002).

The original code by Lara et al. (2011) is conceived to work with a constant water depth $h$ across the whole channel (like in Figure 1, right), i.e. to deal with wet landward conditions only. The possibility to represent the dry landward conditions (Figure 1, left) was achieved by slightly modifying the right boundary condition. In the original code, the following condition for the value of the cross-shore flow velocity $u$ at the $(n-1, j)$-th cell of the numerical domain is imposed :

$u(n-1, j) \propto \frac{h}{h(n-1, j)} \cdot \sqrt{g \cdot h(n-1, j)}$,

where $n$ is the number of the cells in the cross-shore direction $x, j$ is the row-indicator (varying between 1 and the number $m$ of the cells in the vertical direction $z$ ) and $h(n-1, j)$ is the water depth at the $(n-1, j)$-th cell. This condition applies only in case of a free-outflow at the first iteration of each time step.

If $h(n-1, j)=0$, also $u(n-1, j)$ should be 0 ; however, in the original code it tends to $\infty$, resulting in numerical instability and crashing of the simulation.

In addition, if $h(n-1, j)=0$ but $h(n-2, j)>0, u(n-1, j)$ should not be 0 , because this would represent a totally-reflective boundary. Therefore, the boundary condition was modified as follows:

If $h(n-1, j)>0, u(n-1, j) \propto \frac{h}{h(n-1, j)} \cdot \sqrt{g \cdot h(n-1, j)}$, else $u(n-1, j)=u(n-2, j)$.

This algorithm does not affect the original condition for the wave absorption since it applies only when $h(n-1, j)=0$, i.e. before the waves reach the boundary.

The validation of the modified numerical code is provided in Sub-section 2.3.

\subsection{The numerical tests}

The modified version of the IH-2VOF code was used to carry out a series of simulations of irregular waves characterized by Jonswap spectrum against a trapezoidal dike in a twodimensional flume, $43 \mathrm{~m}$ long and $2.0 \mathrm{~m}$ deep. The computational domain has been discretized with a constant mesh of $\Delta z=0.01 \mathrm{~m}$ in the vertical direction and a variable mesh for the crossshore direction. The highest resolution in the $\mathrm{x}$-direction $\Delta \mathrm{x}=0.01 \mathrm{~m}$ is constant in the area around the structure and gradually decreases towards the left and the right boundary sections up to approximately $\Delta x=0.04 \mathrm{~m}$. Overall, the mesh consists of 1733 and 201 cells in the $x$-direction and the z-direction respectively. The layout of the numerical channel is displayed in Figure 2.

For all the simulations, 69 wave gauges (see Figure 2) were installed in the numerical flume to measure the free surface elevations, the pressures and the velocities in front of, over and behind the structure to describe the wave run-up, reflection, overtopping and transmission. In particular the measurements from 5 wave gauges have been used in this paper, and specifically: 
- 3 wave gauges to estimate the wave reflection coefficient $K_{r}$, following the method proposed by Zelt and Skjelbreia (1992). These gauges were placed at approximately $19 \mathrm{~m}$ from the wave generator. The distance from the wave generator and the inter-distances among the gauges $(0.5 \mathrm{~m}$ and $0.1 \mathrm{~m})$ were kept constant during the tests, but accounting for the different wave periods;

- 1 wave gauge at the crest off-shore edge to evaluate the average overtopping discharge $q$;

- 1 wave gauge behind the structure to measure the transmitted wave height and the piling-up.

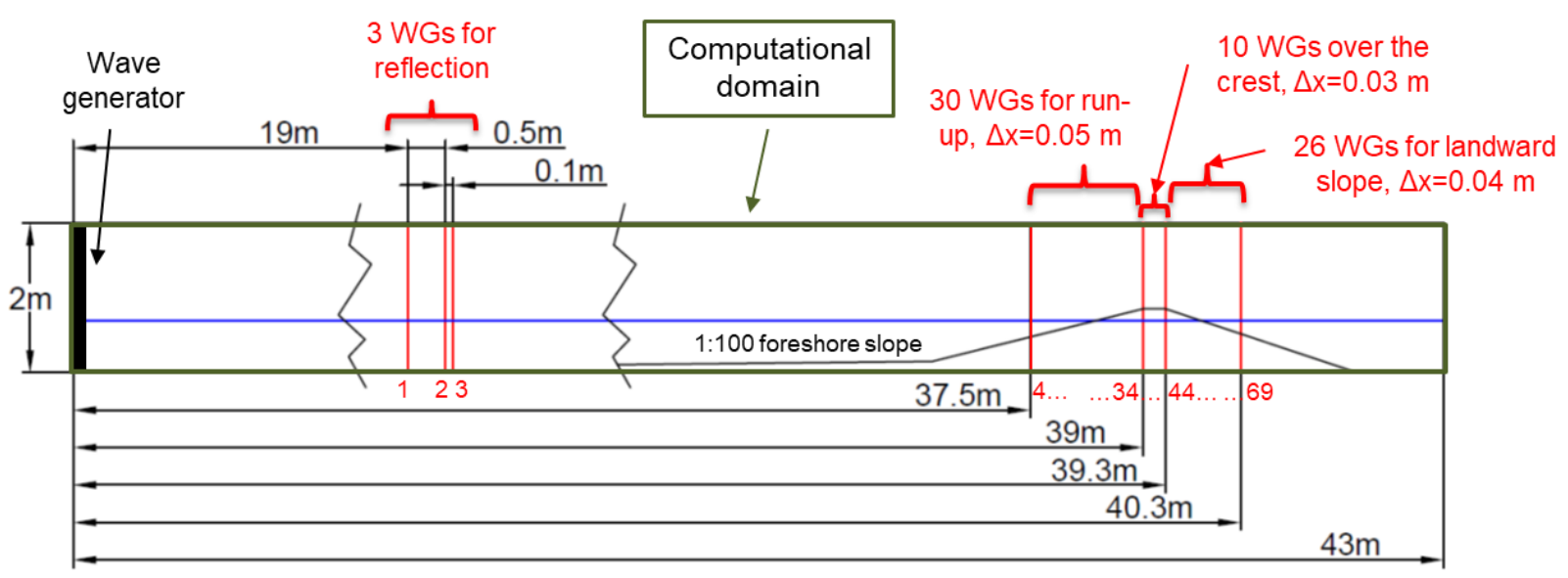

Figure 2- Layout of the numerical flume and wage gauges.

The numerical simulations were carried out on smooth and impermeable structures exclusively. This choice was made for the following reasons:

- the roughness would enhance the wave energy dissipation occurring along the off-shore slope and the permeability would generate filtration, resulting in a reduction of the total overtopping discharge over the crest; the simulation of smooth structures leads therefore to a more cautious approach.

- The introduction of the roughness and of the permeability factors in the numerical model involves the representation of the filtration motion in the porous media, which increases the complexity of the model and the number of parameters to be calibrated (e.g., the coefficients of the Forchheimer's equation, etc.), raising the uncertainty level. Furthermore, the calibration phase should be repeated for each of the values of the roughness factor $\gamma_{f}$ suggested in the literature and would require a systematic investigation that is out of the focus of the present work.

The trapezoidal smooth structure - characterized by varying seaward slope ( $\cot \alpha_{\text {off }}=4$ or 6 ) and fixed landward slope $\left(\cot \alpha_{i n}=3\right)$ - was placed at a distance of $39 \mathrm{~m}$ from the numerical wave generator, see the layout in Figure 2. The crest width $\left(G_{c}\right)$ and the height $\left(h_{c}\right)$ of the structure were kept constant and respectively equal to $0.3 \mathrm{~m}$ and $0.85 \mathrm{~m}$, while its crest free-board $\left(R_{c}\right)$ has been made varying with the still water depth $(h)$. A 1:100 sloping foreshore was located in front of the structure to allow deep water wave generation. 
The dikes were subjected to a variety of irregular wave attacks, with significant wave heights $H_{s}$ equal to 0.1 or $0.2 \mathrm{~m}$ and peak periods $T_{p}$ ranging approximately from 1.3 to $6.5 \mathrm{~s}$. The values of $H_{s}$ and $T_{p}$ were such to determine wave steepnesses $H_{s} / L_{m-1,0}$ varying between 0.02 and 0.05 . The geometrical dimensions, the slopes and the wave attacks were chosen to reproduce "typical" cases of real structures. In particular, we referred to (and extended) the experiments of wave overtopping at seadikes carried out by Schüttrumpf \& Oumeraci (2005) in the small wave flume of the Leichtweiss Institute for Hydraulic Engineering of the Technical University of Braunschweig in Germany.

A free outflow boundary condition was set at the right boundary of the numerical channel. Two different landward conditions were considered for the structure:

- "wet" landward area, to investigate the potential effects of the piling-up and the return flow in case of breached structures;

- "dry" landward area, for the representation of the wave overtopping process in presence of emerged and over-washed dikes.

The scheme of Figure 1 displays the meaning of the "wet" and "dry" landward condition, which basically refers to the presence or absence of water also behind the structures. A "dry" condition applies to over-washed structures, while the "wet" condition applies to breached dikes.

By combining different geometries, wave attacks and landward conditions, a total of 94 tests were carried out. The summary of the target conditions adopted for the tests is reported in Table 1. Not all the possible combinations have been tested. In particular, the dry landward condition was applied only to submerged and zero-freeboard structures, based on the assumption that the return-flow is negligible (or null) in presence of emerged structures. Such assumption was indeed verified by carrying out twice one emerged test at $R_{\delta} / H_{s}=+0.5$ with the same wave conditions $\left(H_{s}=0.1 \mathrm{~m}, H_{s} / L_{m-1,0}=0.03\right)$ and cross-section $\left(\cot \left(\alpha_{o f f}\right)=4\right)$, and varying the landward condition only. The outcomes of the two simulations (wet and dry) were completely identical, both considering the wave overtopping discharge and the flow characteristics above the structure crest (velocity, pressure, depth).

The computational effort necessary to simulate each test was approximately of 3 days for the dikes in dry landward conditions and 1.5 day for the wet conditions. Overall, the set-up of the complete database required nearly $3 \mathrm{~Tb}$ of rough data ( $\approx 32 \mathrm{~Gb}$ for each test). 
Table 1. Summary of the tested conditions with the numerical model. The cases with "dry" landward conditions were all carried out with $H_{s}=0.2 \mathrm{~m}$ and $H_{s} / L_{m-1,0}=0.02$ and 0.03 only.

\begin{tabular}{|c|c|c|c|c|c|c|c|c|}
\hline $\boldsymbol{R}_{\boldsymbol{c}} / \boldsymbol{H}_{\boldsymbol{s}}$ & -1.5 & -1 & -0.5 & -0.2 & 0 & +0.5 & +1 & +1.5 \\
\hline $\boldsymbol{H}_{\boldsymbol{s}} / \boldsymbol{L}_{\boldsymbol{m}-1,0}$ & $0.02 ; 0.03$ & $\begin{array}{c}0.02 ; 0.03 ; \\
0.04\end{array}$ & $\begin{array}{c}0.02 ; 0.03 ; \\
0.05\end{array}$ & 0.02 & $\begin{array}{c}0.02 ; 0.03 ; \\
0.04 ; 0.05\end{array}$ & $\begin{array}{c}0.02 ; 0.03 ; \\
0.04\end{array}$ & $\begin{array}{c}0.02 ; 0.03 ; \\
0.05\end{array}$ & 0.03 \\
\hline $\boldsymbol{H}_{\boldsymbol{s}}[\mathrm{m}]$ & $0.1 ; 0.2$ & $0.1 ; 0.2$ & $0.1 ; 0.2$ & 0.2 & $0.1 ; 0.2$ & $0.1 ; 0.2$ & 0.2 & 0.2 \\
\hline $\boldsymbol{c o t}\left(\boldsymbol{\alpha}_{\text {off }}\right)$ & $4 ; 6$ & $4 ; 6$ & $4 ; 6$ & $4 ; 6$ & $4 ; 6$ & $4 ; 6$ & $4 ; 6$ & $4 ; 6$ \\
\hline $\boldsymbol{c o t}\left(\boldsymbol{\alpha}_{\text {in }}\right)$ & 3 & 3 & 3 & 3 & 3 & 3 & 3 & 3 \\
\hline $\boldsymbol{G}_{\boldsymbol{c}}[\mathrm{m}]$ & 0.3 & 0.3 & 0.3 & 0.3 & 0.3 & 0.3 & 0.3 & 0.3 \\
\hline Landward & wet; dry & wet; dry & wet; dry & dry & wet; dry & wet; dry & wet & wet \\
\hline Tot. \# & 12 & 18 & 18 & 2 & 20 & 16 & 6 & 2 \\
\hline
\end{tabular}

\subsection{Validation of the modified numerical model}

The original IH-2VOF code has been already validated and successfully adopted for modelling the wave overtopping at rubble mound breakwaters (Losada et al., 2005), smooth dikes and seawalls (Reeve et al., 2008; Peng \& Zou, 2011) and harbor caissons (a.o. Misra et al., 2011). The modified code is validated for the first time in this contribution against laboratory tests on wave overtopping at over-washed dikes in dry landward conditions (Sub-section 2.3.1) and at smooth submerged breakwaters in wet landward conditions (Sub-section 2.3.2).

\subsubsection{Validation of the model in over-washed conditions}

The validation of the modified numerical code has been carried out by selecting and reproducing 5 laboratory tests on wave overtopping and overflow against a trapezoidal levee at negative freeboard and dry landward conditions. The selected tests belong to the set of experiments conducted in the wave flume at the U.S. Army Engineer Research and Development Center (ERDC), Coastal and Hydraulics Laboratory (CHL) in Vicksburg, MS (Hughes \& Nadal; 2009; Hughes \& Shaw, 2011), which represent so far the only experience on over-washed dikes available in the literature. The tested levee was characterized by a fixed geometry $\left(\cot \left(\alpha_{\text {off }}\right)=4.25\right.$, $\left.\cot \left(\alpha_{i n}\right)=3, G_{c}=0.122 \mathrm{~m}\right)$ subjected to a variety of wave attacks $\left(H_{s}\right.$ and $\left.T_{p}\right)$ and still water level $(h)$, realizing different crest freeboards $\left(R_{c}\right)$. The detailed description of the experiments is given in Hughes and Nadal (2009), while the structural and hydraulic parameters of the 5 selected tests are reported in model scale units in Table 2 . The water surface elevation registered at the wave gauge in front of the wave maker (wave gauge 1 in Hughes \& Nadal, 2009) were used to force the left-boundary condition in the numerical code.

Table 2 compares also the resulting values of $q$ and $K_{r}$ derived from the numerical simulations of the 5 tests and the corresponding lab measurements (for the tests R18 and R20 the measure of $K_{r}$ was not available). The percentage errors among numerical and experimental values is included between $-23.3 \%$ and +12.5 in case of $q$ and between -12.5 and $+22.2 \%$ in case of $K_{r}$. These percentages and the standard deviations ( $\sigma=0.03$ for $K_{r}$ and $\sigma=0.008 \mathrm{~m}^{3} /(\mathrm{sm})$ for $q$ ) fall in 
the range of the uncertainty associated to the most common predicting method, see Muttray et al. (2006) and Zanuttigh \& van der Meer (2008) for $K_{r}$, and EurOtop (2016) for $q$. Furthermore, no systematic tendency of overestimation or underestimation is observed for both the quantities. These outcomes quantify the accuracy of the modified numerical code and provide a validation of its results in dry landward conditions.

Table 2. Summary of the 5 tests selected from Hughes \& Nadal (2009) and reproduced in the numerical code, and comparison among lab measurements and corresponding numerical estimates of $q$ and $K_{r}$. Lab scale values (1:25).

\begin{tabular}{|c|c|c|c|c|c|c|c|c|c|c|}
\hline \multicolumn{4}{|c|}{$\begin{array}{c}\text { Tests parameters } \\
\text { (model units) }\end{array}$} & \multicolumn{3}{c|}{$q\left[\mathrm{~m}^{3} /(\mathbf{s m})\right]$} & \multicolumn{3}{c|}{$K_{r}$} \\
\hline Test ID & $H_{s}[\mathrm{~m}]$ & $T_{p}[\mathrm{~s}]$ & $R_{c}[\mathrm{~m}]$ & $h[\mathrm{~m}]$ & Lab & Num & $\frac{(\text { Num-Lab) }}{\text { Lab }}$ & Lab & Num & $\frac{(\text { Lab-Num) }}{\text { Lab }}$ \\
\hline $\mathrm{R} 14$ & 0.071 & 2.09 & -0.0110 & 0.621 & 0.0065 & 0.0066 & $1.5 \%$ & 0.27 & 0.31 & $22.2 \%$ \\
\hline $\mathrm{R} 18$ & 0.100 & 2.77 & -0.043 & 0.653 & 0.0202 & 0.0155 & $-23.3 \%$ & - & 0.29 & - \\
\hline $\mathrm{R} 20$ & 0.065 & 2.00 & -0.063 & 0.673 & 0.0285 & 0.0234 & $-17.9 \%$ & - & 0.24 & - \\
\hline $\mathrm{R} 109$ & 0.099 & 2.73 & -0.012 & 0.622 & 0.0056 & 0.0063 & $12.5 \%$ & 0.35 & 0.34 & $-2.9 \%$ \\
\hline $\mathrm{R} 118$ & 0.097 & 2.37 & -0.032 & 0.642 & 0.0121 & 0.0118 & $-2.5 \%$ & 0.32 & 0.28 & $-12.5 \%$ \\
\hline
\end{tabular}

\subsubsection{Validation of the model in overtopping and breached conditions}

In the literature, very few data on wave overtopping at breached and smooth dikes are available. Most of the experience on submerged structures is indeed focused on the analysis of $K_{r}$ and $K_{t}$ (Van der Meer et al., 2005). To our knowledge, the only tests available to validate the numerical code in breached conditions are the $2 \mathrm{D}$ experiments on wave overtopping at a submerged plywood breakwater carried out by Cox \& Tajziehchi (2005) in the wave flume of the Water Research Laboratory of the University of New South Wales, Australia. These tests consist of a set of different combinations of 12 monochromatic waves against 3 different trapezoidal breakwaters at negative freeboard $\left(R_{c}=-0.05,-0.1\right.$ and $\left.-0.15 \mathrm{~m}\right)$, characterized by 3 crest widths $\left(G_{c}=3.5,1.5\right.$ and $\left.0.3 \mathrm{~m}\right)$ and fixed slopes $\left(\cot \left(\alpha_{\text {off }}\right)=\cot \left(\alpha_{i n}\right)=2\right)$. The 12 monochromatic waves include both breaking and non-breaking conditions, with values of the wave steepness $H / L$ varying approximately in the range [0.01-0.07] and values of $H / h$ in the range [0.01-0.5]. Further information about the experiments and the details of the experimental setup, the tested conditions and the measured values of $q$ can be found in Tajziehchi (2006).

The tests for the validation have been selected by considering the geometrical and hydraulic features of the numerical tests (see Table 1). Specifically, we selected the breakwater characterized by $G_{c}=0.3 \mathrm{~m}$ (tests "t3", in Tajziehchi, 2006) and a set of 5 waves so that the values of $H / L$ and $R_{d} / H$ were similar to the wave conditions realized within the new numerical tests (Section 2.2). Each experimental test has been reproduced in the numerical code by simulating 
$50 \mathrm{~s}$ of wave attack (corresponding to a number of 25-50 waves according to the wave period $\mathrm{T})$. The characteristics of the 5 selected tests, the corresponding measurements and numerical values of $q$ are here reported in Table 3.

Also in this case the percentage errors among numerical and experimental values are modest, being in the range $[-9.3 \% ;+6.9]$, with a standard deviation $\sigma=0.004 \mathrm{~m}^{3} /(\mathrm{sm})$. The errors have a random distribution. These outcomes quantify the accuracy of the modified numerical code and provide a validation of its results in wet landward conditions.

Table 3. Summary of the 5 tests selected from Tajziehchi (2006) and reproduced in the numerical code, and comparison among lab measurements and corresponding numerical estimates of $q$. Lab scale values.

\begin{tabular}{|c|c|c|c|c|c|c|c|c|}
\hline \multicolumn{6}{|c|}{ Tests parameters } & \multicolumn{3}{|c|}{$q\left[\mathrm{~m}^{3} /(\mathrm{sm})\right]$} \\
\hline Test ID & $\begin{array}{c}H \\
{[\mathrm{~m}]}\end{array}$ & $\begin{array}{c}T \\
{[\mathrm{~s}]}\end{array}$ & $\begin{array}{l}R_{c} \\
{[\mathrm{~m}]}\end{array}$ & $\begin{array}{c}h \\
{[\mathrm{~m}]}\end{array}$ & $\begin{array}{c}H / L \\
{[-]}\end{array}$ & Lab & Num & $\frac{\text { (Num-Lab) }}{\text { Lab }}$ \\
\hline t3-21 & 0.058 & 1.00 & -0.1 & 0.40 & $3.9 \%$ & 0.0043 & 0.0039 & $-9.30 \%$ \\
\hline t3-30 & 0.118 & 2.00 & -0.1 & 0.40 & $3.0 \%$ & 0.0146 & 0.0152 & $4.11 \%$ \\
\hline t3-42 & 0.115 & 1.49 & -0.15 & 0.45 & $4.0 \%$ & 0.0115 & 0.0123 & $6.96 \%$ \\
\hline $\mathrm{t} 3-46$ & 0.091 & 2.00 & -0.15 & 0.45 & $2.2 \%$ & 0.0092 & 0.0087 & $-5.43 \%$ \\
\hline t3-47 & 0.147 & 2.00 & -0.15 & 0.45 & $3.6 \%$ & 0.0147 & 0.0135 & $-8.16 \%$ \\
\hline
\end{tabular}

\subsection{Preliminary verification of the numerical tests}

This Sub-section aims at verifying the results of the numerical simulations in terms of $K_{r}$ and $K_{t}$ values against experimental data and against the predictions by the Artificial Neural Network (ANN) tool developed by Zanuttigh et al. (2016) and Formentin et al. (2017) and adopted by EurOtop (2016).

Considering the tested conditions used for the numerical simulations, the experimental data available for comparison are:

- $K_{r}$ : tests on smooth straight slopes $\left(\operatorname{cota}_{\text {off }}=[0.7 ; 6]\right)$, carried out in wave flume and dry landward conditions at $R_{d} / H_{s}>0$ (Zanuttigh \& Van der Meer, 2008);

- $K_{r}$ and $K_{t}$ : tests on smooth LCS, carried out in wave basin and wet landward conditions, and characterized by $G_{c} / H_{s}=[0 ; 8.6], R_{c} / H_{s}=[-8.0 ; 1.3]$, cota ${ }_{\text {off }}=[1.5 ; 4]$ (Van der Meer et al., 2005).

Besides these tests, if one considers similar structures, the ANN has been trained also on a number of permeable LCS data in wave basin and in wave flume (Kramer et al., 2005).

The comparison with the experimental data is provided in Figures 3-a and 3-b, while the comparison with the ANN predictions is given in Figures 4-a and 4-b for $K_{r}$ and $K_{t}$ respectively. 
The diagrams relative to $K_{r}$ include both the dry and the wet numerical tests, as it is expected that the structure landward conditions only marginally affect the wave reflection and therefore no particular difference should be observed between the two conditions. This guess is indeed confirmed by Figure 4-a, that shows no bias or heteroscedasticity related to the landward conditions among ANN predictions and numerical values. The comparison for $K_{t}$ is instead given for the wet tests only (Figures 3-b and 4-b) as no transmitted wave height can be computed for tests in dry conditions.
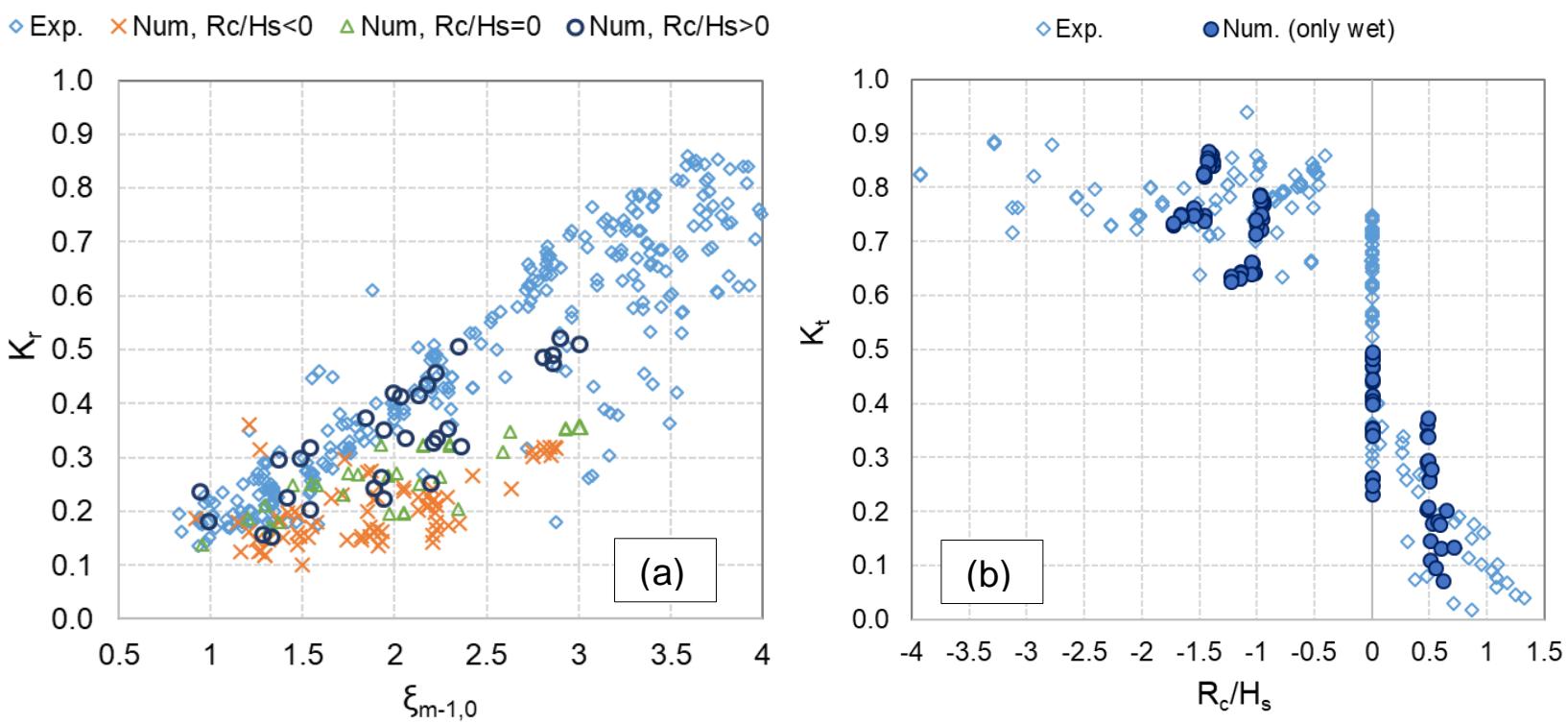

Figure 3 - Values of $K_{r}$ (panel a) and $K_{t}$ (panel b) computed from the numerical simulations compared to the corresponding experimental values available for smooth and impermeable slopes (a: Zanuttigh \& Van der Meer, 2008; b: Van der Meer et al., 2005). The $K_{r}$-values are shown as functions of $\xi_{m-1,0}$ and are grouped in classes of $R_{c} / H_{s}$. The $K_{t}$-values are shown as functions of $R_{d} / H_{s}$. In panel a both the wet and dry numerical tests are shown, while in panel b only the wet tests are shown. 

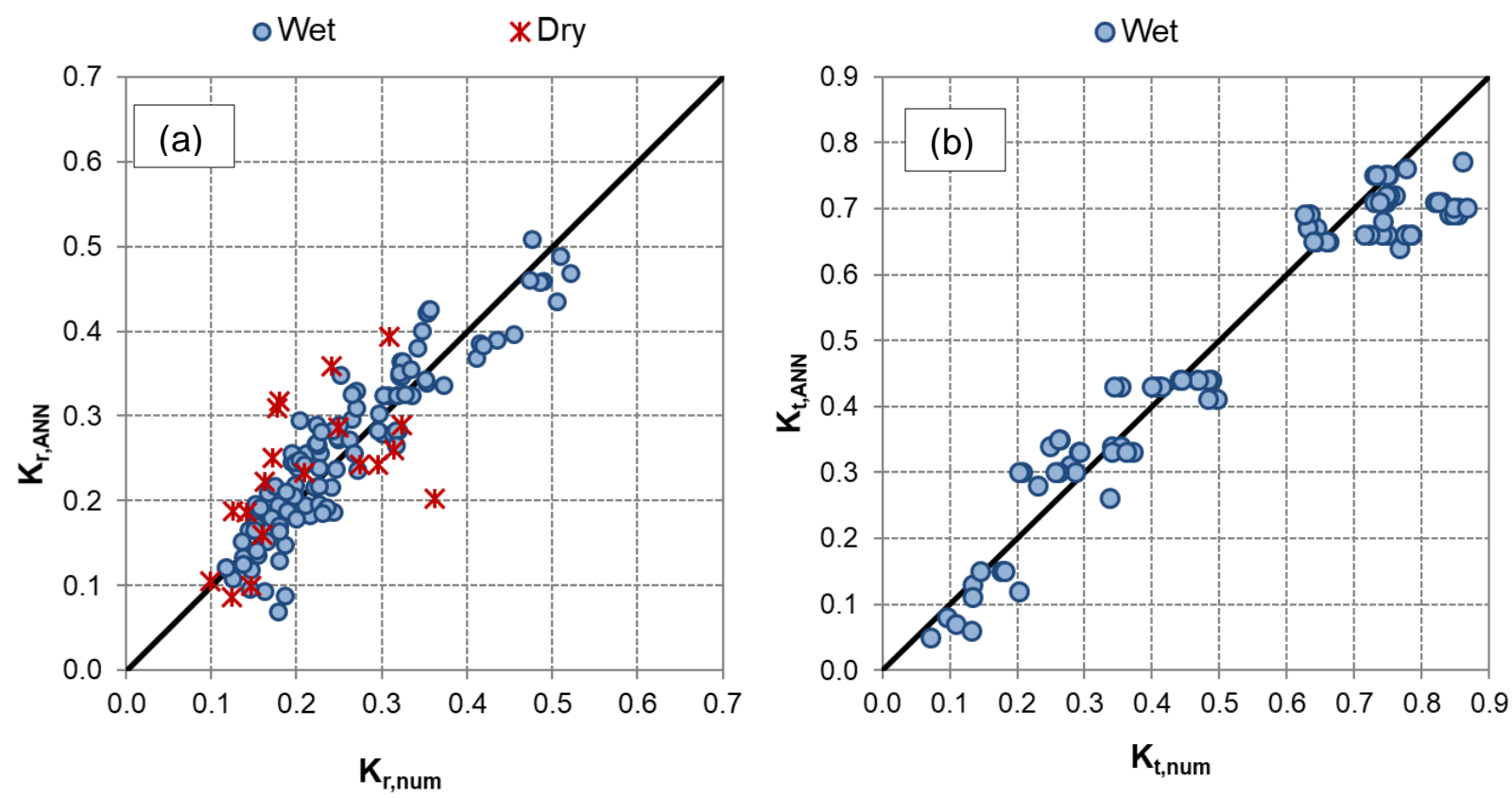

Figure 4 - Values of $K_{r}$ (a-panel) and $K_{t}$ (b-panel) computed from the numerical simulations (respectively, $K_{r}$, num and $K_{t, \text { num }}$ ) compared to the corresponding values predicted with the ANN by Formentin et al., 2017 (respectively, $K_{r, A N N}$ and $K_{t, A N N}$ ). In a-panel, both the wet and dry tests are shown, while in panel-b only the wet tests are shown.

Figures 3-a,b show that both the numerical values of $K_{r}$ and $K_{t}$ follow the same trends of the experimental data against $\xi_{m-1,0}$ and $R_{d} / H_{s}$, respectively The numerical values of $K_{t}$ are all included in the cloud of the experimental data, while part of the numerical $K_{r}$-values are lower than the corresponding experimental ones. These data consistently correspond to structures at $R_{c} / H_{s} \leq 0$ (see Figure $3-a$ ), which induce less wave reflection than the emerged structures characterizing the lab data.

The agreement among numerical results and ANN predictions (Figure 4) is quantitatively represented by the values of $\sigma=0.06$ and $R^{2}=0.88$ in case of $K_{r}$ and of $\sigma=0.08$ and $R^{2}=0.96$ in case of $K_{t}$. These values are particularly satisfactory if compared to the average optimal performance of the ANN tool achieved when predicting the data used for its training (i.e. calibration), which gives $\sigma \approx 0.035$ for both $K_{r}$ and $K_{t}$ and $\mathrm{R}^{2}=0.96-0.99$ for $K_{r}$ and $K_{t}$ respectively (see Formentin et al., 2017).

In conclusion, the combined analysis of Figures 3 and 4 demonstrates that the numerical model gives a reliable and consistent representation of $K_{r}$ and $K_{t}$ in both dry and wet landward conditions. This proves in turn that the model can accurately reproduce the wave overtopping process, since reflection, overtopping and transmission are strictly correlated, i.e. the lower the reflection the higher the run-up, the overtopping and the transmission. 


\section{Limits of the existing methods}

Aim of this Section is to compare the numerical results with the existing formulae for the prediction of $q$. These formulae have been traditionally developed separately for emerged and zero-freeboard structures $\left(R_{c} / H_{s} \geq 0\right)$ or for negative freeboards $\left(R_{c} / H_{s}<0\right)$. All the formulae for $R_{c} / H_{s}<0$ are targeted to represent dry structures only. Therefore the numerical results are compared to the corresponding formulae in two separate Sub-sections, 3.1 and 3.2 for $R_{d} / H_{s}>0$ and $R_{c} / H_{s}<0$ respectively. The effects of the wet landward conditions are presented and discussed in Sub-section 3.3, investigating the reasons of the inadequacy of the formulae for the representation of fully-breached or submerged structures.

Note that the EurOtop ANN tool (Zanuttigh et al., 2016) for the prediction of $q$ is not adopted for comparison since it was calibrated exclusively on structures in dry landward conditions with $R_{c} / H_{s} \geq 0$. Therefore most of the new tests presented here are out of the field of validity of this method.

\subsection{Emerged and zero-freeboard structures}

In case of $R_{d} / H_{s} \geq 0$ the value of $q$ can be predicted by means of the well-consolidated formulae provided by the EurOtop manual (2016). For a probabilistic design these formulae give:

$q_{\text {overtop }}=\min \left\{\begin{array}{l}\frac{0.023}{\sqrt{\tan \alpha}} \cdot \xi_{m-1,0} \cdot \exp \left(-\left(2.7 \frac{R_{c}}{\xi_{m-1,0} \cdot H_{s} \cdot V_{b} \cdot V_{f} \cdot V_{v} \cdot V_{\beta}}\right)^{1.3}\right) \cdot \sqrt{g H_{s}^{3}} \\ 0.09 \cdot \exp \left(-\left(1.5 \frac{R_{c}}{H_{s} \cdot \gamma_{f} \gamma_{\beta}}\right)^{1.3}\right) \cdot \sqrt{g H_{s}^{3}}\end{array}\right.$

where $\gamma_{b}, \gamma_{f}, \gamma_{v}$ and $\gamma_{b}$ are the influence factors for a berm, roughness elements on a slope, a crown wall and oblique wave attacks. In the case of the numerical tests presented in this paper, all these factors are equal to 1.

Figure 5 compares the numerical values of $q\left(q_{\text {num }}\right)$ with the corresponding estimates by Eq. (2) ( $\left.q_{\text {Eur }}\right)$. The comparison is provided in terms of relative errors $\left(q_{\text {Eur }}-q_{\text {num }}\right) / q_{\text {Eur }}$ as functions of $R_{d} / H_{s}$, and the data are grouped by values of $\cot \alpha_{\text {off }}(4$ and 6$)$. The values of $R_{c} / H_{s}$ are here computed from the incident wave heights $H_{s}$ measured at the structure toe instead of the target values reported in Table 1. The values of $q_{\text {num }}$ are of the same order of magnitude of $q_{\text {Eur, }}$ as all the errors fall within a range of $\pm 40 \%$. These percentages are comparable to the errors associated to the EurOtop formulae and to the most common predicting methods, which may often show differences among predictions and measurements greater than the 30\% (EurOtop, 2016). No significant bias of the error is observed neither towards $R_{c} / H_{s}$, nor $\cot \alpha_{\text {off }}$ or other parameters, such as the wave steepness and the breaker parameter (these latter results are not reported for sake of brevity). 


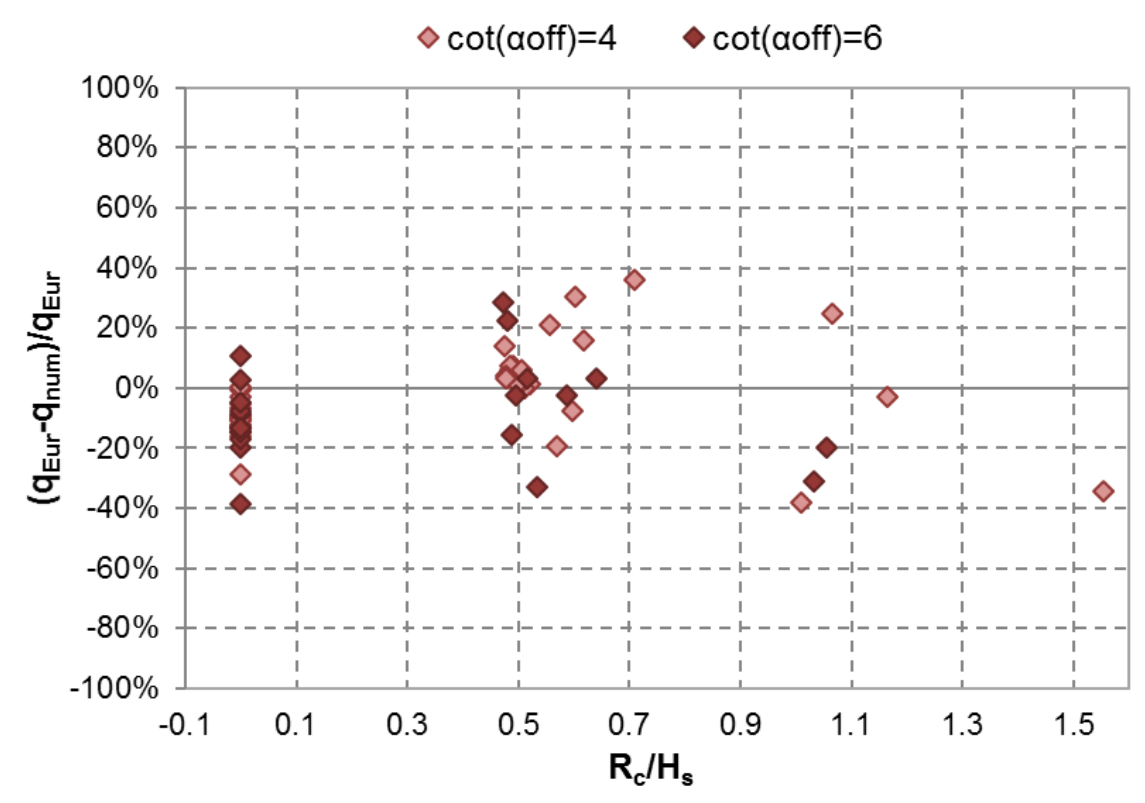

Figure 5 - Relative percentage error $\left(q_{\text {Eur }}-q_{\text {num }}\right) / q_{\text {Eur }}$ among the values of $q$ derived from the numerical code $\left(q_{\text {num }}\right)$ and the corresponding estimates $\left(q_{\text {Eur }}\right)$ by the EurOtop (2016) formulae, as functions of $R_{c} / H_{s}$. The values are grouped according to the value of the off-shore slope ( $\cot \alpha_{\text {off }}$ ) of the structures. Tests at $R_{c} / H_{s} \geq 0$ only.

\subsection{Negative freeboard: over-washed structures}

The reference methods for the prediction of $q$ in case of $R_{c} / H_{s}<0$ are the EurOtop formulae (2016) and the formulae by Hughes \& Nadal (2009). Both these methods are based on the conceptual splitting of the total overtopping into two components: the steady water overflow due to a storm surge higher than the crest freeboard (hereinafter, $q_{\text {overflow) }}$ and the overtopping water due to the incoming waves ( $\left.q_{\text {overtop}}\right)$.

The first component is treated as the flow over a broad-crested weir in open channels, assuming that the dike crest is wide enough and that the friction losses are negligible to ensure a hydrostatic pressure distribution and determine the occurrence of the critical flow at some point over the crest. Under these hypotheses, qoverflow becomes proportional to the upstream head (i.e. the difference between the surge elevation and the dike crest freeboard) through an empirical coefficient. EurOtop (2007), (2016) proposed the use of the simple following equation:

$q_{\text {overflow }}=0.54 \sqrt{g \cdot \mid-R_{c}^{3}} /$

where the upstream head is expressed in term of the negative crest freeboard $R_{c}$ (see Figure 1). The EurOtop manual suggests to simply add the overflow term given by Eq. (3) to the overtopping component $q_{\text {overtop }}$ of Eq. (2), where $R_{c}$ must be set to 0 . 
Hughes \& Nadal (2009) - based on a series of experimental tests - derived the following predictive equation for $q$ :

$q=0.0336 \cdot \sqrt{g H_{m 0}^{3}}+0.973 \cdot q_{\text {overflow }}\left(-\frac{R_{c}}{H_{s i}}\right)^{0.083}$

where $q_{\text {overflow }}$ in this case is computed as

$q_{\text {overflow }}=0.5443 \sqrt{g} \cdot\left(-R_{c}\right)^{\frac{3}{2}}$

This approach still splits $q$ into the two contributes but, differently from the EurOtop formulae, tries to account for the combined effect of storm surge and incoming waves, instead of simply summing them. Note that Eq. (5) is formally identical to Eq. (3), the only (little) difference being the weir coefficient.

Figures 6 and 7 compares the values of $q_{\text {num }}$ with the predictions from EurOtop (2016), $q_{\text {Eur, and }}$ from Hughes and Nadal (2009), $q_{H N}$, respectively. The two figures show very similar trends of the relative errors as functions of $R_{d} / H_{s}$. The values of $q_{\text {num }}$ are systematically overestimated by both formulae, being the lower the $R_{d} / H_{s}$, the higher the overestimation. The greatest difference between the two formulae is observed for $R_{d} / H_{s} \approx-0.2$ (dry tests), where $\left(q_{\text {Eur }} q_{\text {num }}\right) / q_{\text {Eur }} \approx+10 \%$ while $\left(q_{H N}-q_{n u m}\right) / q_{H N} \approx-50 \%$. The better agreement of the EurOtop formulae in this case of modest submergence is justified by the fact that these formulae are targeted to zero-freeboard conditions.

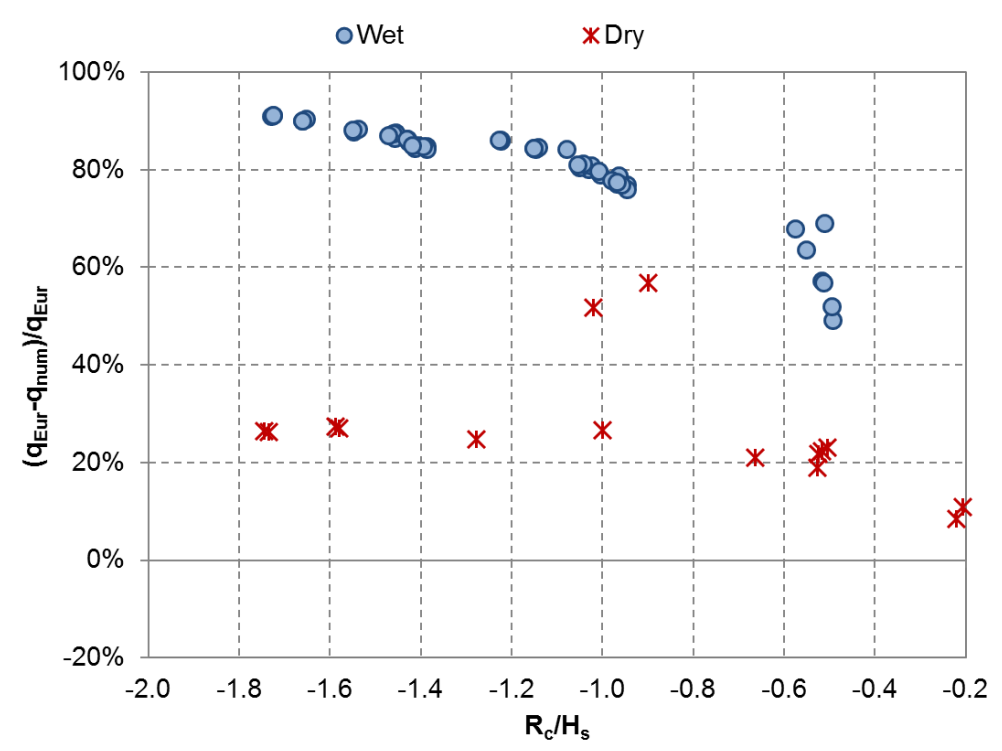

Figure 6 - Relative percentage error $\left(q_{\text {Eur }}-q_{\text {num }}\right) / q_{\text {Eur }}$ among the values of $q$ derived from the numerical code ( $q_{\text {num }}$ ) and the corresponding estimations ( $q_{\text {Eur }}$ ) by the EurOtop (2016) formulae, as functions of $R_{d} / H_{s}$. The values are grouped according to the landward condition (dry or wet). Tests at $R_{c} / H_{s}<0$. 


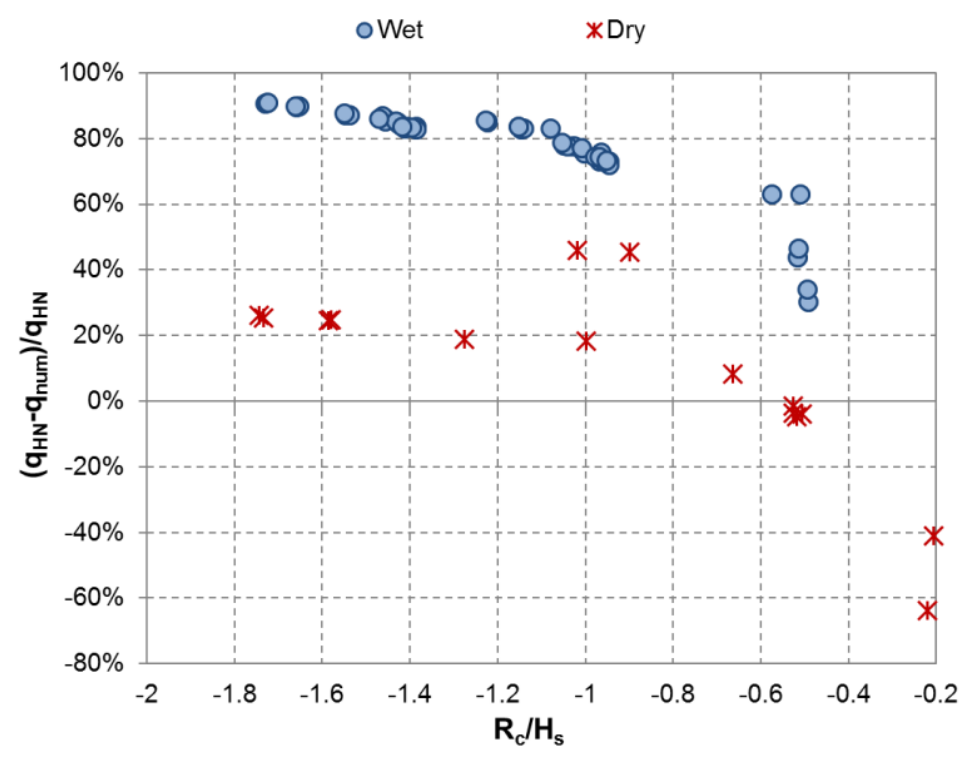

Figure 7 - Relative percentage error $\left(q_{H N}-q_{n u m}\right) / q_{H N}$ among the values of the wave overtopping discharge derived from the numerical code $\left(q_{\text {num }}\right)$ and the corresponding estimations $\left(q_{H N}\right)$ by Hughes and Nadal (2009) formulae, as functions of $R_{c} / H_{s}$. The values are grouped according to the landward condition (dry or wet). Tests at $R_{c} / H_{s}<0$.

The dry tests are sensibly less overestimated by the formulae than the wet tests. With the exception of two outliers around $R_{c} / H_{s} \approx-1$, the dry tests are characterized by an error that is on average equal to $20-25 \%$ and that in Figure 7 drops to 0 when $R_{c} / H_{s} \approx-0.5$. Such errors are comparable or even lower than the errors derived for the tests in emerged conditions (see Figure 5 ). On the contrary, the overestimation of the wet tests is approximately of one order of magnitude in both figures.

\subsection{Why the existing formulae are insufficient}

The results of Figures 6 and 7 indicate that the overtopping is strongly affected by the landward conditions. A qualitative explanation of this phenomenon is provided by Figure 8, which reproduces the instantaneous snapshots of the passage of a wave trough over the structure crest during the simulation of the same submerged test $\left(R_{d} / H_{s}=-1.5\right)$ for an over-washed (a) and a breached (b) structure. In case of over-washing, a nearly constant hydraulic gradient is present between the off-shore and the in-shore sections of the structure crest. After the complete breaching, the presence of water at the landward slope reduces or nullifies such hydraulic gradient. This is particularly evident during the trough phase, when the head over the structure crest of a wet tests may be even lower than the still water level at the off-shore edge. In such conditions, the result is an inversion of the horizontal hydraulic gradient, which induces a returnflow over the dike crest and negative values of the cross-shore flow velocity $u$. 


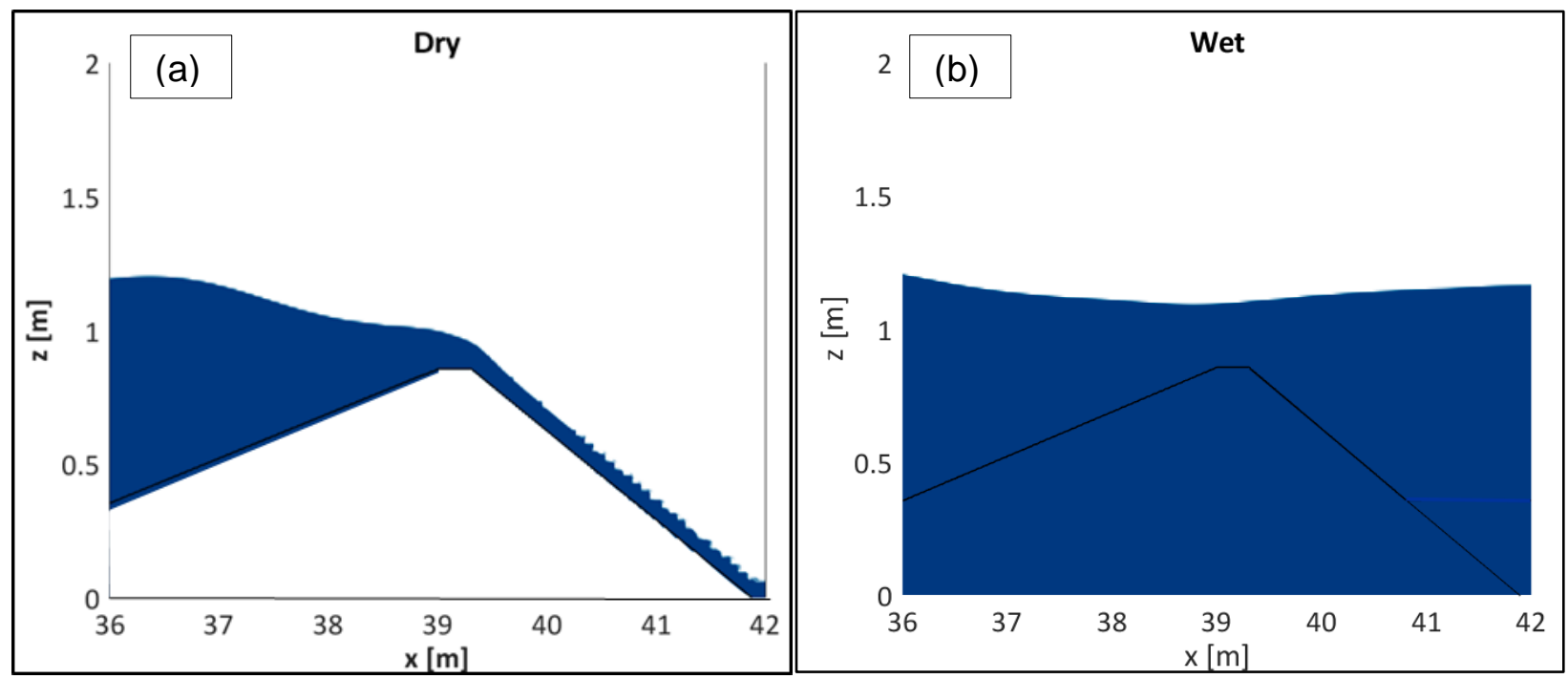

Figure 8 - Instantaneous snapshots of the numerical wave flume in correspondence of the structure during the simulation of the same submerged test $\left(R_{c} / H_{s}=-1.5\right)$ carried out with dry (a) and wet (b) landward conditions. Both the snapshots refer the passage of a wave trough over the structure crest $(x \approx 39 \mathrm{~m})$.

As an example, Figure 9 shows a 5 seconds-time-evolution of the flow depth $(h)$ above the structure crest, for a breached condition with $R_{c}=-0.3 \mathrm{~m}$ and $R_{d} / H_{s}=-1.5$. The vertical profiles of $u(\mathrm{~m} / \mathrm{s})$ registered at the wave crest and at the wave trough ( $\mathrm{t}=1 \mathrm{~s}$ and $\mathrm{t}=3.8 \mathrm{~s}$, respectively) are reported in Figure 10. From Figures 9 and 10, it is clear that the sign of $u$ depends on the wave phase: during the crest phase (Figure 10, a) the flux is in-shore directed along the whole vertical, while during the trough phase (Figure 10, b) the values of $u$ become negative, revealing the presence of an off-shore directed flux (return flow).

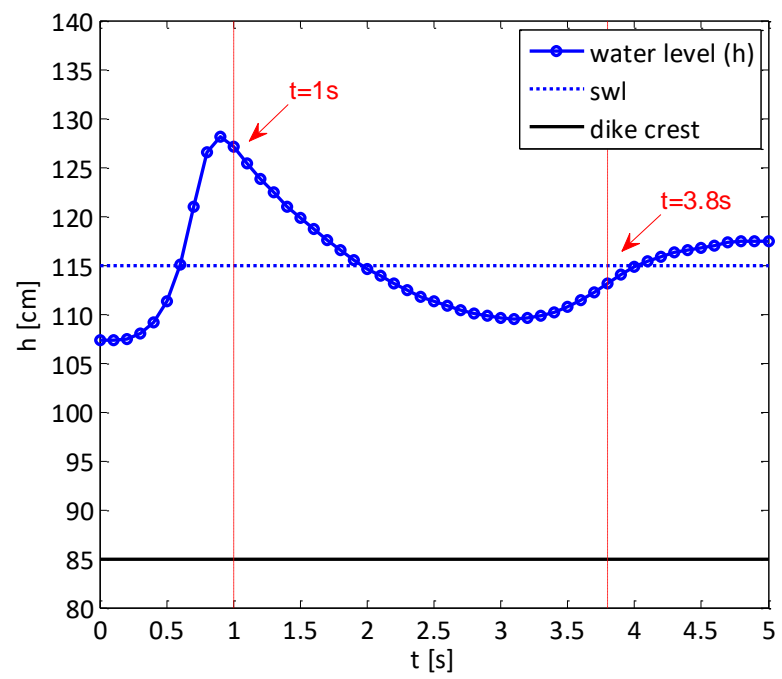

Figure 9 - Time evolution (5 seconds) of the flow depth $(h)$ over the crest of a submerged dike (black line), compared to the still water level (blue dots). 

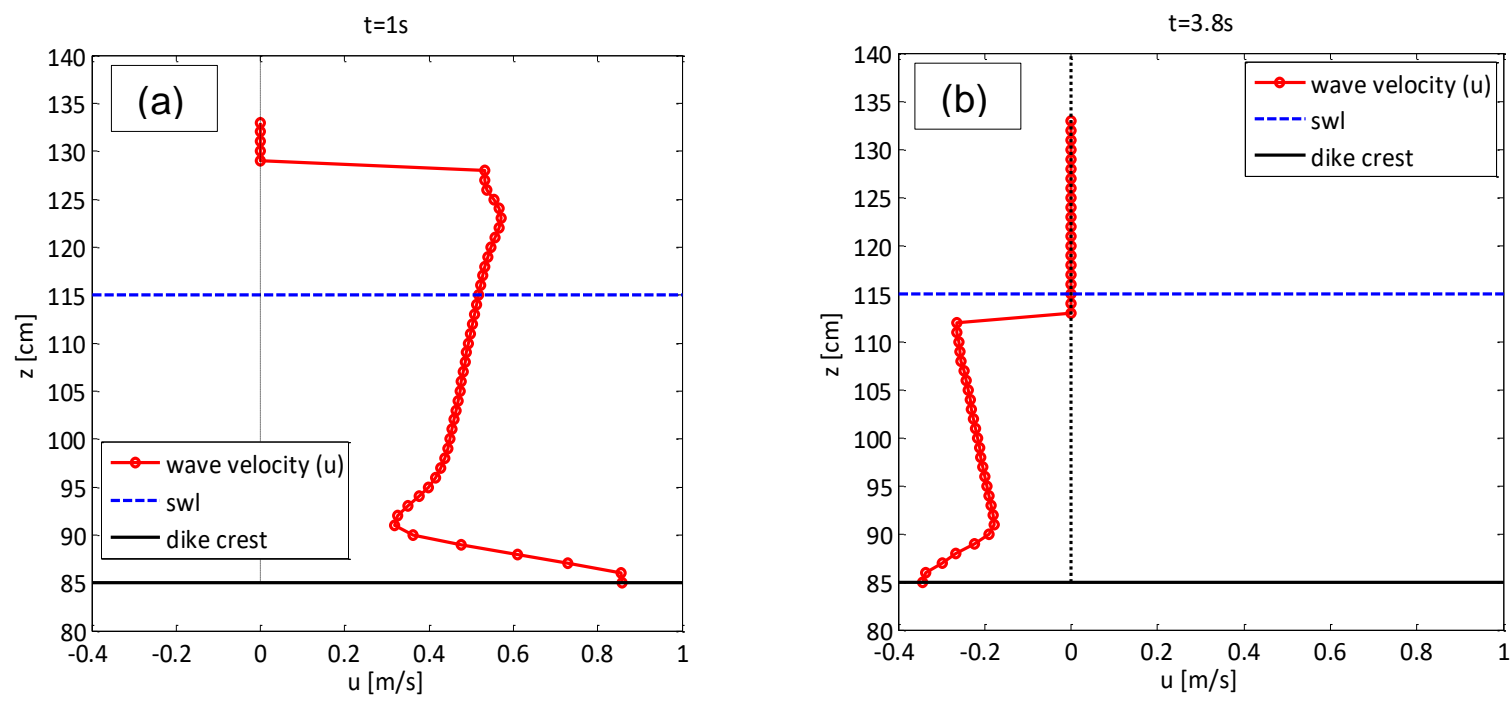

Figure 10 - Vertical profiles $(z)$ of the horizontal component of the flow velocity $(u)$ over the crest of a submerged structure (black line), during the crest phase (a) and the trough phase (b). The still water level is indicated for comparison (blue dots).

The inverse horizontal gradient may be enhanced by the occurrence of piling-up ( $\left.p_{\text {up }}\right)$ behind the structure due to the wave breaking over the structure crest (Zanuttigh et al., 2008). Indeed, nonnegligible $p_{u p}$-values were measured behind the structures in case of wet tests. Figure 11 reports the average values of $p_{u p}$ derived from the numerical wave gauges placed along the landward edge of the structures. The values of $p_{u p}$ are higher in case of $R_{c} / H_{s} \geq-1$, while when $R_{d} / H_{s}<-1 p_{u p}$ tends to zero, in agreement with the results of Zanuttigh et al. (2008).

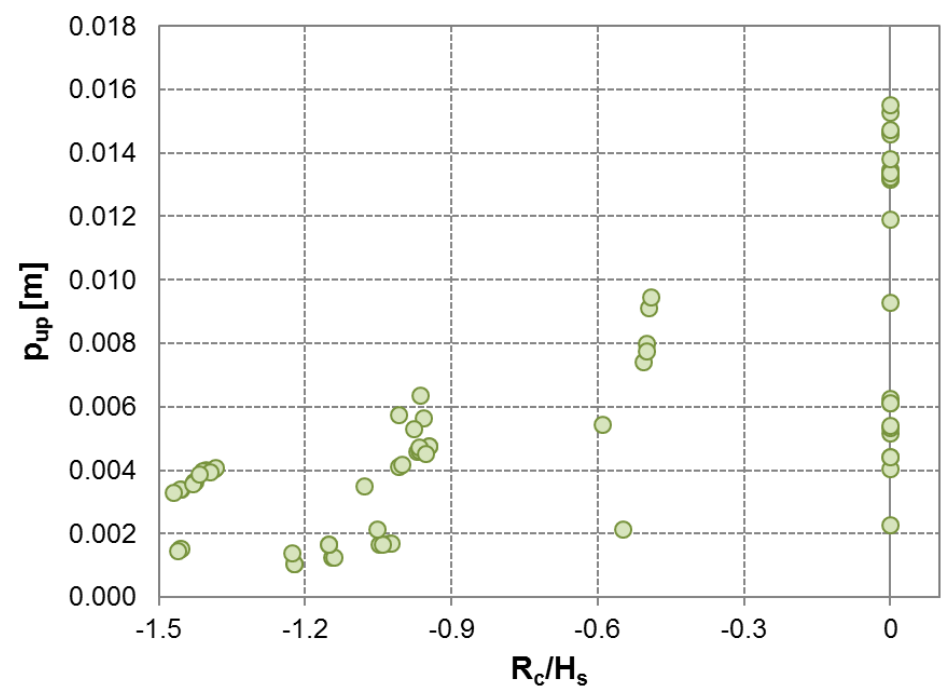

Figure 11 - Values of the piling-up ( $\left.p_{\text {up }}\right)$ measured behind the structures in case of "wet tests" (breached dikes) as functions of $R_{d} / H_{s}$. Negative freeboards only $\left(R_{c} / H_{s}<0\right)$. 
In summary, in case of a breached dike, i.e in wet conditions:

- the horizontal gradient is significantly reduced (with respect to dry conditions) by the presence of water behind the structure, even during the crest wave phase;

- during the trough wave phase, the gradient may be inverted and a return flow may occur;

- the reduction (crest phase) or inversion (trough phase) of the gradient are enhanced by the piling-up.

The combination of these effects leads to a significant reduction of qoverflow that becomes an intermittent flow mainly (or exclusively) governed by the wave pumping, instead than a quasisteady weir-like flow. This can thus explain the overestimation of the values of $q$ given by the formulae (Figures 5 and 6).

\section{The new method for over-washed and breached dikes}

A new conceptual method has been conceived to estimate $q$ at over-washed and breached dikes. Such method starts from the conceptual model developed by Zanuttigh et al. (2008) to represent $q$ and $p_{u p}$ at low crested structures.

Similarly to the current approach (EurOtop, 2016 and Hughes \& Nadal, 2009), the new method is based on the separation of the contributions due to the wave pumping and the overflow. The novelty of the method consists in the set-up of a unique physically based formulation that accounts for $p_{\text {up }}$ but can be used indifferently whether $p_{\text {up }}$ is zero (over-washed dikes) or not (breached dikes).

Despite the model has been calibrated on the numerical tests on smooth dikes, it includes the possibility to be extended to rubble mound and permeable structures, through the introduction of the roughness factor $Y_{f}$, whose effectiveness has been checked in the validation phase.

The equations of the new method are provided in the following.

$$
\begin{aligned}
& q=q_{\text {drift }}+q_{\text {overflow. }}, \quad \text { for } \frac{R_{c}}{H_{s}} \leq 0, \text { where: } \\
& q_{\text {drift }}=\lambda_{s} \cdot \frac{H_{r m s}^{2}}{8} \cdot \frac{c_{s}}{h_{D}}+\lambda_{r} \cdot 0.9 \cdot \frac{H_{r m s}^{2}}{T_{m}}, \\
& q_{\text {overflow }}=a \cdot \exp \left(b \cdot \frac{R_{c}}{V_{f} H_{s}}-\frac{p_{u p}}{H_{r m s}}\right) \cdot\left(\frac{H_{s}}{L_{m-1,0}}\right)^{-\frac{1}{4}} \cdot \sqrt{g \cdot\left(H_{r m s}-R_{c}\right)^{3}}
\end{aligned}
$$

where: $q_{\text {drift }}$ is the drift discharge component due to the wave pumping; $q_{\text {overflow }}$ is the overflow component depending on $R_{c}$ and on $p_{u p}$ in case of wet tests; the values of the coefficients $\lambda_{s}, \lambda_{r}$, and $a, b$ of Eq.s (7) and (8) are reported in Tables 4 and 5, respectively. 
Eq. (7) is directly derived from Zanuttigh et al. (2008) and Calabrese et al. (2005). The description of the procedure for the computation of $q_{d r i f t}$ and the meaning of the physical quantities of Eq. (7) is given in Sub-section 4.1.

The expression of Eq. (8) has been developed by analyzing the existing formulae and the physical processes and by fitting the coefficients on the basis of the results of the numerical simulations. Eq. (8) is proposed in this work for the first time. The details and the physical interpretation of Eq. (8) are provided in Sub-section 4.2.

This new method is described (Sub-section 4.1 and 4.2) and validated against the numerical tests, characterized by $\gamma_{f}=1$ (Sub-section 4.3) in comparison with the existing models (Hughes \& Nadal, 2009 and EurOtop, 2016). The validity of the method is checked on experimental data for overtopped and over-washed dikes ( $\gamma_{f}=1$ and dry landward conditions, Sub-section 5.1) and for breached rubble mound LCS ( $\gamma_{f}=0.4$ and wet landward conditions, Sub-section 5.2) as no data for breached smooth dikes were available.

\subsection{The drift component of the new approach}

Eq. (7) consists of two terms, $\lambda_{s} \cdot \frac{H_{r m s}^{2}}{8} \cdot \frac{c_{s}}{h_{D}}$ and $\lambda_{r} \cdot 0.9 \cdot \frac{H_{r m s}^{2}}{T_{m}}$, representing the Stokes and the roller mass drift, respectively. According to the literature (Zanuttigh et al., 2008), both the calibration factors $\lambda_{s}$ and $\lambda_{r}$ are here kept equal to 1 (see Table 4). $T_{m}$ is the mean wave period and $H_{r m s}$ is the root-mean-squared wave height computed as the geometric mean between the transmitted $\left(H_{r m s, t}\right)$ and the incident $\left(H_{r m s, i}\right)$ wave heights:

$H_{r m s}=\sqrt{H_{r m s, i} \cdot H_{r m s, t}}=\sqrt{\frac{H_{s i}}{1.4} \cdot \frac{H_{s t}}{1.4}}$

For the computation of the transmitted wave height $H_{s t}$, it is suggested to derive the wave transmission coefficient $K_{t}$ from the consolidated formulae of Van der Meer et al. (2005) for the case of smooth structures.

In Eq. (7), $c_{s}$ is the wave celerity over the structure crest, that can be computed as $c_{s}=\sqrt{g h_{D}}$ (see Table 4) under the hypothesis of shallow water linear theory. The term $h_{d}=h_{m}+0.5 \cdot H_{r m s}$ is the average water depth over the structure crest accounting for the wave pumping, where $h_{m}$ is the average water depth over the crest between the wave breaking point and the breaking end (that coincides with the in-shore edge of the dike crest, see the scheme in Figure 12.

The value of $h_{m}$ is computed as:

$h_{m}=h_{m o}+\frac{p_{u p}}{2}, \quad h_{m o}=h-\left(h_{c}-\frac{h_{b}+R_{c}}{2 \cdot\left(G_{c}+x_{b}\right)} \cdot x_{b}\right)$.

The terms $h_{b}$ and $x_{b}$ are, respectively, the breaking depth and the distance between the breaking 
point and the seaward crest edge (see Figure 12), and are estimated by the following formulae by Calabrese et al. (2003):

$h_{b}=h\left(\frac{H_{s i}}{r_{b} h}\right)^{\frac{4}{5}} ; \quad x_{b}=\frac{h_{b}+R_{c}}{\tan \alpha_{o f f}}$

where $\gamma_{b}$ is the breaker index (Battjes and Janssen, 1978) and it is set to 0.6 in this work (see Table 4). If no piling-up occurs (dry tests, $\left.p_{u p}=0\right), h_{m}=h_{m o}$ in Eq. (10) and can be directly used to derive $h_{D}$ that appears in Eq. (7). Otherwise, $h_{m}$ depends on the value of $p_{u p}$, and it can be derived (Zanuttigh et al., 2008) from the resolution of the cross-shore momentum equation applied to a control volume around the dike cross-section included between the breaking starting and the breaking end point (i.e. the area where the piling-up takes place). The selected control volume is drawn in Figure 12 with red dashed-dotted lines.

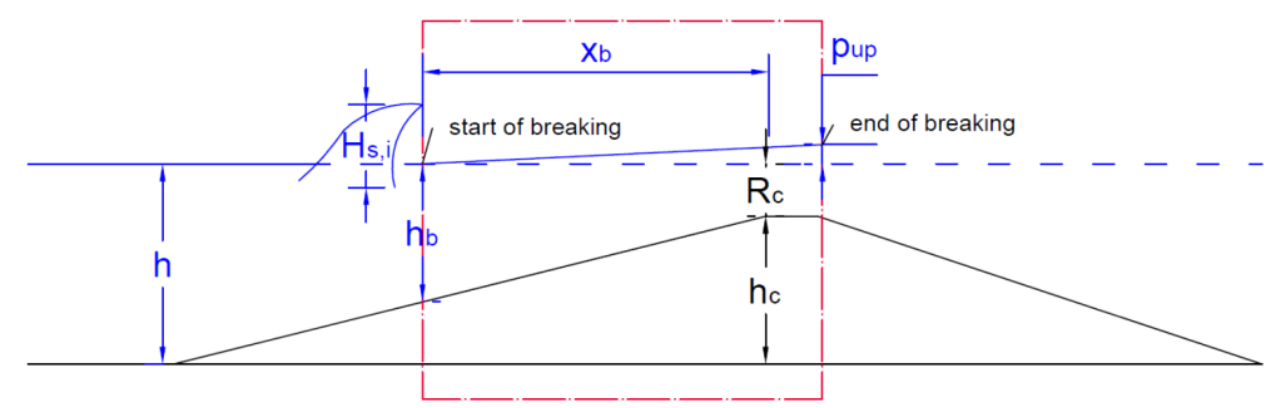

Figure 12 - Scheme of the dike cross-section and the control volume (dashed-dotted red lines) for the cross-shore momentum equation and the evaluation of the piling-up.

The cross-shore momentum can be written in the following simplified form:

$\bar{\Pi}+\bar{S}+\bar{R}=0$

that accounts only for the resultant pressure force on the control volume surface $(\bar{\Pi})$, the resultant momentum excess due to the waves (the radiation stress $\bar{S}$ ) and the resultant of the friction force over the crest dike $(\bar{R})$. The terms related to the momentum excess due to the currents $(\bar{C})$ and to the filtration $(\bar{F})$ are both null, because of the $2 \mathrm{D}$ domain $(\bar{C}=0)$ and the impermeability of the dikes $(\bar{F}=0)$.

The terms $\bar{\Pi}$ and $\bar{S}$ of Eq. (12a) are formulated as:

$\bar{\Pi}=\left[-\rho g p_{u p}\left(h_{m o}+\frac{p_{u p}}{2}\right)\right] ; \bar{S}=\left[\frac{1}{16} \rho g\left(H_{s i}^{2}-H_{s t}^{2}\right) \cdot\left(\frac{1}{2}+\frac{2 k h}{\sinh (2 k h)}\right)\right]$

Formally, Eq. (12a,b) should account also for the contribution of $\bar{R}$, since a slipping boundary condition was assumed at the fluid-obstacle interface (see Sub-section 2.2). Based on Zanuttigh et al. (2008) and Calabrese et al. (2003), $\bar{R}$ is a function of the undertow seaward-directed 
discharge $q_{u}$ and of the $p_{u p}$ itself, hence the inclusion of $\bar{R}$ leads to a $5^{\text {th }}$-degree equation in $p_{u p}$, which could be solved only numerically. Yet, it has been verified by the authors that the friction contribution is negligible for the smooth structure surfaces. In this cases, $\bar{R}$ is at least one order of magnitude lower than $\bar{\Pi}$ and two orders of magnitude lower than $\bar{S}$. The error made by computing $p_{u p}$ with $\bar{R}=0$ is lower than the $1 \%$. In case of rubble-mound breakwaters $\left(\gamma_{<}<1\right)$, the effect of $\bar{R}$ is not negligible, being on average $\bar{R} / \bar{\Pi} \approx 0.4$ (Calabrese et al., 2005). To keep the application of this new method simple and practical, the effects of the friction are represented by the inclusion of the $\gamma_{f}$ coefficient in Eq. (8). The validity of this assumption is checked against experimental data on rubble mound LCS (Sub-section 5.2).

Therefore, Eq. (12) can be rewritten as the following $2^{\text {nd }}$ order equation in $p_{\text {up }}$ :

$p_{u p}^{2}+2 p_{u p} h_{m o}-\frac{2}{\rho g} S=0 \leftrightarrow p_{u p}=-h_{m o} \pm \sqrt{h_{m o}^{2}+\frac{2 S}{\rho g}}$

Table 4. Values/expression of the coefficients adopted for the computation of $q_{\text {drift }}$ and $q$ (Eq.s 7 and 11).

\begin{tabular}{|c|c|}
\hline Parameter & Adopted value/expression \\
\hline$\lambda_{s}$ & 1 \\
\hline$\lambda_{r}$ & 1 \\
\hline$T_{m}$ & $\approx T_{p} / 1.24 \cdot$ \\
\hline$h_{D}$ & $h_{m}+0.5 \cdot H_{r m s}$ \\
\hline$c_{s}$ & $\sqrt{g h_{D}}$ \\
\hline$\gamma_{b}$ & 0.6 \\
\hline
\end{tabular}

\subsection{The overflow component of the new approach}

Eq. (8) is composed by the 3 factors that are physically involved in the overflow process:

- the weir-like flow due to the average head over the structure crest;

- the return flow over the crest induced by the $p_{u p}$;

- the wave breaking process at the toe of the structure induced by the wave steepness.

The contribution of the weir-like flow depends on the average head over the structure crest due to the wave pumping and is represented by the term $\left(H_{r m s}-R_{c}\right)$, accounting for wave transmission.

From the experimental results (see Figure 11), the values of $p_{u p}$ decrease with increasing submergence, therefore the lower the $R_{d} / H_{s}$, the lower the $p_{u p}$. The higher the $p_{u p}$, the higher the return flow and therefore the lower qoverflow. The result of the combined effects of $p_{u p}$ and $R_{c} / H_{s}<0$ on $q_{\text {overflow }}$ is expressed in Eq. (8) as $\exp \left(b \frac{R_{c}}{\gamma_{f} H_{s, i}}-\frac{p_{u p}}{H_{r m s}}\right)$. This factor reproduces the same 
exponential relationship between $q$ and $R_{d} / H_{s}$ of the EurOtop formulae for emerged tests (Eq. 2), but introduces two keys elements - the coefficient $b$ and $-\frac{p_{u p}}{H_{r m s}}-$ which allow to account for the different dynamics of the overtopping process among dry and wet conditions $(b)$ and for the return flow induced by $p_{u p}$.

The sign of $b$ has been made varying with the landward conditions (see Table 5 ) in order to reflect the physics of the process:

- in case of dry tests, the more submerged the structure, the higher the hydraulic gradient between the off-shore and the in-shore cross sections of the dike crest and therefore the

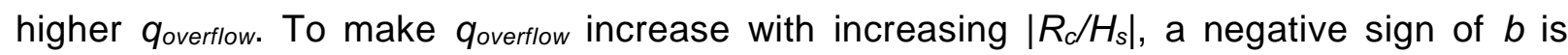
necessary.

- on the contrary, for wet tests, the more submerged the structure, the less effective the qoverflow component, as the total overtopping rate tends to coincide with the drift overtopping component. The result is a positive sign of $b$ that makes qoverflow decreases with increasing $\left|R_{c} / H_{s}\right|$.

The numerical values of $b$ have been derived from the fitting of the numerical data. The value of $b=-0.35$ for the dry tests induces a much steeper variation of $q_{\text {overflow }}$ with $R_{c} / H_{s}$ than the value of $b=1.2$ for the wet tests. The very mild decrease of $q_{\text {overflow with }} R_{d} / H_{s}$ nearly results into a constant relationship (see the example application of Section 6).

Following the form of Eq. (2), the effects of a rough structure surface are represented by the $1 / \gamma_{f}$ coefficient, which is included in the exponential term multiplying $R_{d} / H_{s}$, similarly to EurOtop formulae (Eq.s (2)+(3)).

The $p_{u p}$-term applies only to the wet tests because $p_{u p}=0$ for the dry tests and therefore $\exp \left(-\frac{p_{u p}}{H_{r m s}}\right)=1$. The introduction of $\frac{p_{u p}}{H_{r m s}}$ in the exponential function allows to keep the same expression for the dry and for the wet tests, contemporarily accounting for the inherent dependency of $p_{u p}$ on $R_{d} / H_{s}$. Note that $p_{u p}$ is made dimensionless with the average wave height over the structure crest $H_{r m s}$, which is the governing parameter of the piling-up process.

The effects of breaking due to wave steepness are included in the term $\left(\frac{H_{s, i}}{L_{m-1,0, t}}\right)^{\frac{1}{4}}$, where $L_{m-1,0}$ is here computed on the basis of the spectral wave period $T_{m-1,0}\left(L_{m-1,0}=1.56 \cdot T_{m-1,0, t}^{2}\right)$ and $H_{s, i}$ is the wave height measured in the flume.

The dependence of $q_{\text {overflow }}$ on these 3 physical contributions can be expressed in the same way for both dry and wet conditions. The only difference is the coefficient $b$ multiplying $R_{c} / H_{s}$ that is negative for the dry and positive for the wet conditions (see Table 5). 
Table 5. Values of the coefficients adopted for the computation of qoverflow (Eq. 8).

\begin{tabular}{|c|c|}
\hline Parameter & Adopted value \\
\hline$a$ & 0.055, for both dry and wet tests \\
\hline$b$ & $\left\{\begin{array}{l}-0.35, \text { dry tests }\left(p_{u p}=0\right) \\
1.2, \text { wet tests }\left(p_{u p} \neq 0\right)\end{array}\right.$ \\
\hline
\end{tabular}

\subsection{Results and performance on the numerical data}

The results of the application of the new method given by Eq.s (6)-(7)-(8) to the numerical tests are shown in the following, from both a qualitative and quantitative point of view.

Figure 13 provides a qualitative indication of the relative errors among the numerical data $\left(q_{\text {num }}\right)$ and the corresponding computations of the new formulae $\left(q_{\text {new }}\right)$. In the Figure, the values are plotted as functions of $R_{c} / H_{s}$ and are grouped according to the landward condition. Differently from Figures 5 and 6, Figure 13 includes also the tests at $R_{c} / H_{s}=0$. Figure 13 indicates that the error $\left(q_{\text {new }}-q_{\text {num }}\right) / q_{\text {new }}$ is always included between $\pm 20 \%$. Such value is comparable or even lower than the average errors associated to the EurOtop formulae applied to emerged tests (Figure 4). However, the errors in Figure 13 are more symmetrically distributed around the zero if compared to Figure 4, both considering the dry and the wet tests. A slight bias of the error is detected in case of wet tests: all the data tend to be overestimated (though the relative error is $<20 \%$ ) for $R_{c} / H_{s}$ in the interval [-1.3;-1]. The greatest scatter is observed at $R_{c} / H_{s}=0$.

The relative values of the standard deviation ( $\sigma \%$, normalized with respect to the average values of $q$ of the datastes) and of the coefficient of determination $\left(R^{2}\right)$ characterizing the agreement among $q_{\text {new }}$ and $q_{\text {num }}$ are given in Table 6 . The performance indices associated to the wet and the dry tests $\left(\sigma \%=8 \%\right.$ and $12 \%$ and $R^{2}=0.96-0.99$, respectively) result vey similar, revealing that the new method can represent the 2 typologies of landward conditions with approximately the same accuracy. In Table 6 , the values of $\sigma$ and $R^{2}$ associated to the predictions of the dry tests are provided also for $q_{\text {Eur }}$ and $q_{H N}$ (i.e. the estimations of $q_{\text {num }}$ given by the EurOtop, 2016 and the Hughes \& Nadal, 2009 formulae, respectively). The comparison suggests that the numerical dry tests are better represented by the new method than by the existing formulae, being the $\sigma$ equal or higher than $40 \%$ for $q_{E u r}$ and $q_{H N}$ (instead of $11 \%$ for $q_{\text {new }}$ ) and $R^{2}$ respectively equal to 0.54 and 0.64 (with respect to 0.98 for $q_{\text {new) }}$ ). 


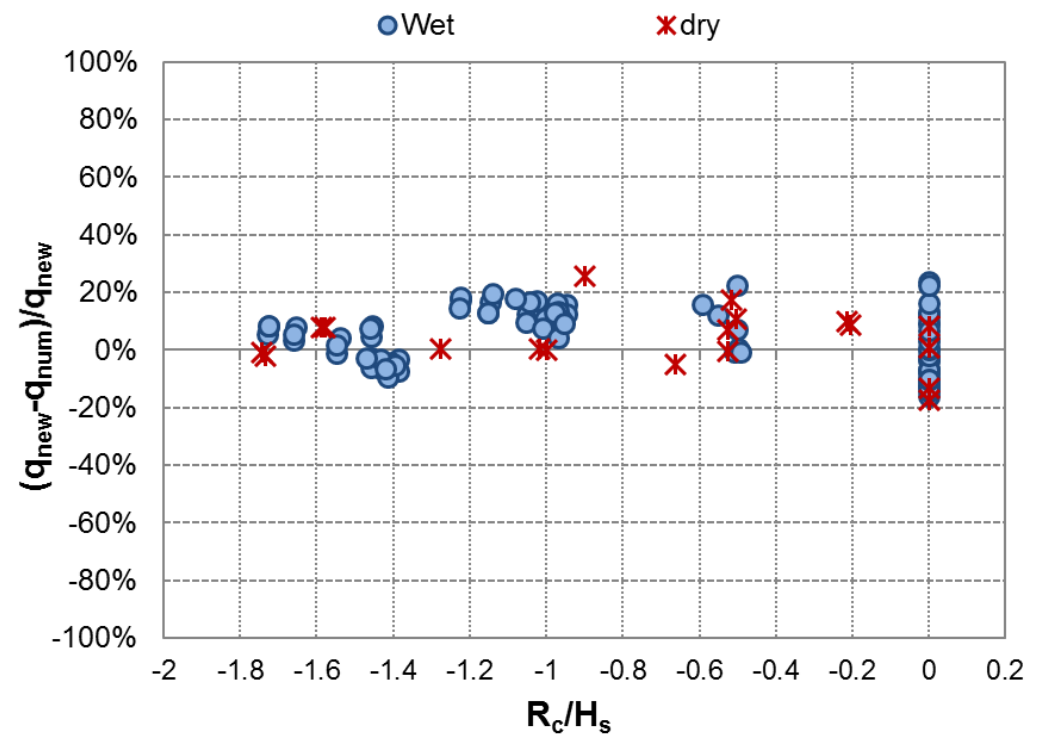

Figure 13 - Relative percentage errors $\left(q_{\text {new }}-q_{\text {num }}\right) / q_{\text {new }}$ among the values of $q$ derived from the numerical code $\left(q_{\text {num }}\right)$ and the corresponding estimations $\left(q_{\text {new }}\right)$ by the new method provided by Eq.s (6)-(7)-(8), as functions of $R_{c} / H_{s}$. The values are grouped according to the landward condition. Tests at $R_{c} / H_{s} \leq 0$ only.

Table 6. Values of the percentage standard deviations $(\sigma \%)$ and of the coefficients of determination $\left(R^{2}\right)$ associated to the estimations of the numerical/experimental tests given by: the new model (Eq.s 6-7-8); the formulae by Hughes \& Nadal, 2009 (Eq.s 4-5); the EurOtop formulae (Eq.s 2-3).

\begin{tabular}{|c|c|c|c|c|c|c|c|}
\hline \multicolumn{2}{|c|}{ Dataset } & \multicolumn{2}{c|}{ Eq.s (6)-(7)-(8) } & \multicolumn{2}{c|}{ Eq.s (4) and (5) } & \multicolumn{2}{c|}{ Eq.s (2)+(3) } \\
\cline { 2 - 8 } \multicolumn{2}{|c|}{} & $\sigma \%$ & $R^{2}[-]$ & $\sigma \%$ & $R^{2}[-]$ & $\sigma \%$ & $R^{2}[-]$ \\
\hline \multirow{2}{*}{$\begin{array}{c}\text { Numerical } \\
\text { data used for } \\
\text { calibration }\end{array}$} & Dry tests & $8 \%$ & 0.99 & $44 \%$ & 0.64 & $50 \%$ & 0.54 \\
\cline { 2 - 8 } & Wet tests & $12 \%$ & 0.96 & - & - & - & - \\
\hline \multirow{4}{*}{$\begin{array}{c}\text { Experimental } \\
\text { datasets for } \\
\text { validation }\end{array}$} & $\mathrm{O}$ (dry) & $17 \%$ & 0.91 & - & - & $11 \%$ & 0.95 \\
\cline { 2 - 8 } & $\mathrm{HN}$ (dry) & $27 \%$ & 0.80 & $9 \%$ & 0.98 & $29 \%$ & 0.76 \\
\cline { 2 - 8 } & $\mathrm{SC}$ (dry) & $12 \%$ & 0.83 & - & - & $16 \%$ & 0.72 \\
\cline { 2 - 8 } & $\mathrm{FI}-1$ (wet) & $33 \%$ & 0.85 & - & - & - & - \\
\cline { 2 - 8 } & $\mathrm{FI}$ (wet) & $43 \%$ & 0.95 & - & - & - & - \\
\cline { 2 - 8 } & $\mathrm{BAU}($ wet) & $13 \%$ & 1.00 & - & - & - & - \\
\hline
\end{tabular}




\section{Verification of the new method on experimental data}

In this Section the new method is verified on experimental data. Since the method is conceived to work for both wet and dry landward conditions, two different typologies of experiments available from the literature have been selected accordingly. The first typology (Sub-section 5.1) refers to tests on overtopped and over-washed dikes in dry landward conditions, similar to the numerical tests used for calibration. The second typology of experiments (Sub-section 5.2) refers to rubble mound LCS, as no measurement of $q$ is available for breached dikes, viz smooth structures in wet landward conditions. This second application has been carried out by setting the value of $\gamma_{f}=0.4$ in Eq. (8), checking thus also the effectiveness of including of $\gamma_{f}$ in place of $\bar{R}$ (see Sub-section 4.1) as term to account for the effects due to friction in case of rough structure surfaces.

\subsection{Overtopped and over-washed dikes}

The accuracy of the new method for dry tests has been verified by computing $q_{\text {new }}$ for 3 sets of laboratory measurements on wave overtopping against smooth slopes $\left(\gamma_{f}=1, \cot \alpha_{\text {off }}>0\right)$ at $R_{c} / H_{s} \leq 0$ and dry landward condition. The following 3 datasets have been selected from the literature:

- the 27 tests on levees $\left(R_{c} / H_{s}=[-0.1 ;-2.0]\right.$, cota $\left.\alpha_{\text {off }}=4.25, G_{c}=3.05 \mathrm{~m}\right)$ performed by Hughes and Nadal, 2009 (dataset HN, hereinafter), and used to derive Eq.s (4) and (5);

- 8 tests on smooth dikes at $R_{d} / H_{s}=0$ from the database of Owen, 1980 (dataset OW, hereinafter);

- 12 tests on smooth dikes ( $\cot \alpha_{\text {off }}=3$ or $4, G_{c}=0$ ) at $R_{d} / H_{s}=0$ from Schüttrumpf, 2001 (dataset $\mathrm{SC}$, hereinafter);

The results of the application are quantitatively reported in Table 6 in terms of $\sigma$ and $R^{2}$ among $q_{\text {new }}$ and the corresponding measured values $q_{\text {meas }}$ for the 3 datasets, and are qualitatively shown in Figures 14 and 15. Figure 14 compares the values of $q_{\text {new }}$ to $q_{\text {meas }}$ and $q_{\text {num }}$ (for the laboratory and numerical tests, respectively), while Figure 15 shows the percentage errors $\left(q_{\text {new }}-q_{\text {meas }}\right) / q_{\text {new }}$ as functions of $R_{d} / H_{s}$. The performance of $q_{\text {new }}$ can be compared with $q_{H N}$ (when applicable) and $q_{\text {Eur }}$ in terms of $\sigma$ and $R^{2}$ in Table 4 and of the relative errors $\left(q_{\text {Eur }}-q_{\text {meas }}\right) / q_{\text {Eur }}$ and $\left(q_{H N}-q_{\text {meas }}\right) / q_{H N}$ in Figure 15.

In case of the lab datasets, the indexes in Table 6 suggest that the performance of $q_{\text {new }}$ is on average comparable with $q_{\text {Eur, }}$, being, respectively the best and the worst cases $R^{2}=0.91, \sigma=22 \%$ (dataset OW) and $R^{2}=0.80, \sigma=27 \%$ (dataset HN) for $q_{\text {new }}$ and $R^{2}=0.95, \sigma=11 \%$ (dataset OW) and $R^{2}=0.72, \sigma=16 \%$ (dataset SC) for $q_{\text {Eur. Both }} q_{\text {new }}$ and $q_{\text {Eur }}$ are characterized by a similar performance ( $R^{2}=0.80, \sigma=27 \%$ and $R^{2}=0.76, \sigma=29 \%$, respectively) when applied to the $\mathrm{HN}$ data. As expected, this dataset is significantly better represented by $q_{H N}\left(R^{2}=0.98, \sigma=9 \%\right)$. The performance of $q_{H N}$ against the OW and SC data is not given since $q_{H N}$ cannot be applied to these datasets characterized by $R_{c} / H_{s}=0$. 


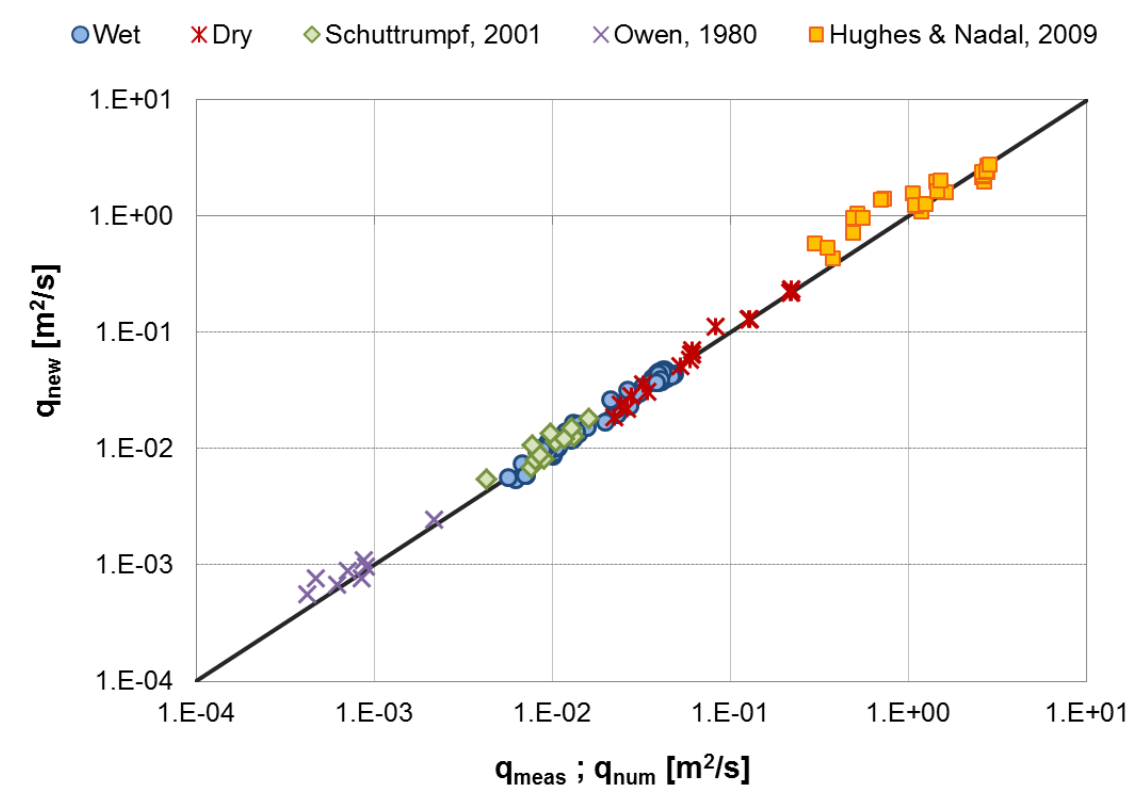

Figure 14 - Comparison among the values of $q$ computed with the new method $\left(q_{\text {new }}\right)$ and the corresponding measured/numerical values $\left(q_{\text {meas }} ; q_{\text {num }}\right)$ for 3 selected dataset of laboratory tests on smooth over-washed dikes and for the numerical simulations.

Figure 14 gives a qualitative idea of the agreement among $q_{\text {new }}$ and experimental data $\left(q_{\text {meas }}\right)$ and among $q_{\text {new }}$ and the numerical data $\left(q_{\text {num }}\right)$. The $\mathrm{HN}$ dataset is very well represented when $q_{\text {meas }}>1 \mathrm{~m}^{2} / \mathrm{s}$, while the lower values of $q_{\text {meas }}$ ( 8 tests in total) are overestimated by $q_{\text {new. }}$ From Figure 15 (a), it can be appreciated that these data correspond to the condition of $R_{c} / H_{s}>-0.4$, where $\left(q_{\text {new }}-q_{\text {meas }}\right) / q_{\text {new }}$ is on average the $40-50 \%$. With the exception of such tests from HN and 1 test from OW, $\left(q_{\text {new }}-q_{\text {meas }}\right) / q_{\text {new }}$ is always included between $\pm 35 \%$. The error is distribution is not biased, and the greatest scatter is concentrated around $R_{d} / H_{s}=0$.

A similar scatter at $R_{c} / H_{s}=0$ is observed for $q_{\text {Eur }}$ (Figure 15, c) that, however, tends to systematically overestimate all the $\mathrm{HN}$ tests and the numerical dry tests. In addition, 2 tests of SC are significantly underestimated by $q_{\text {Eur, }}$, being $\left(q_{\text {Eur }}-q_{\text {meas }}\right) / q_{\text {Eur }} \approx 50 \%$. 

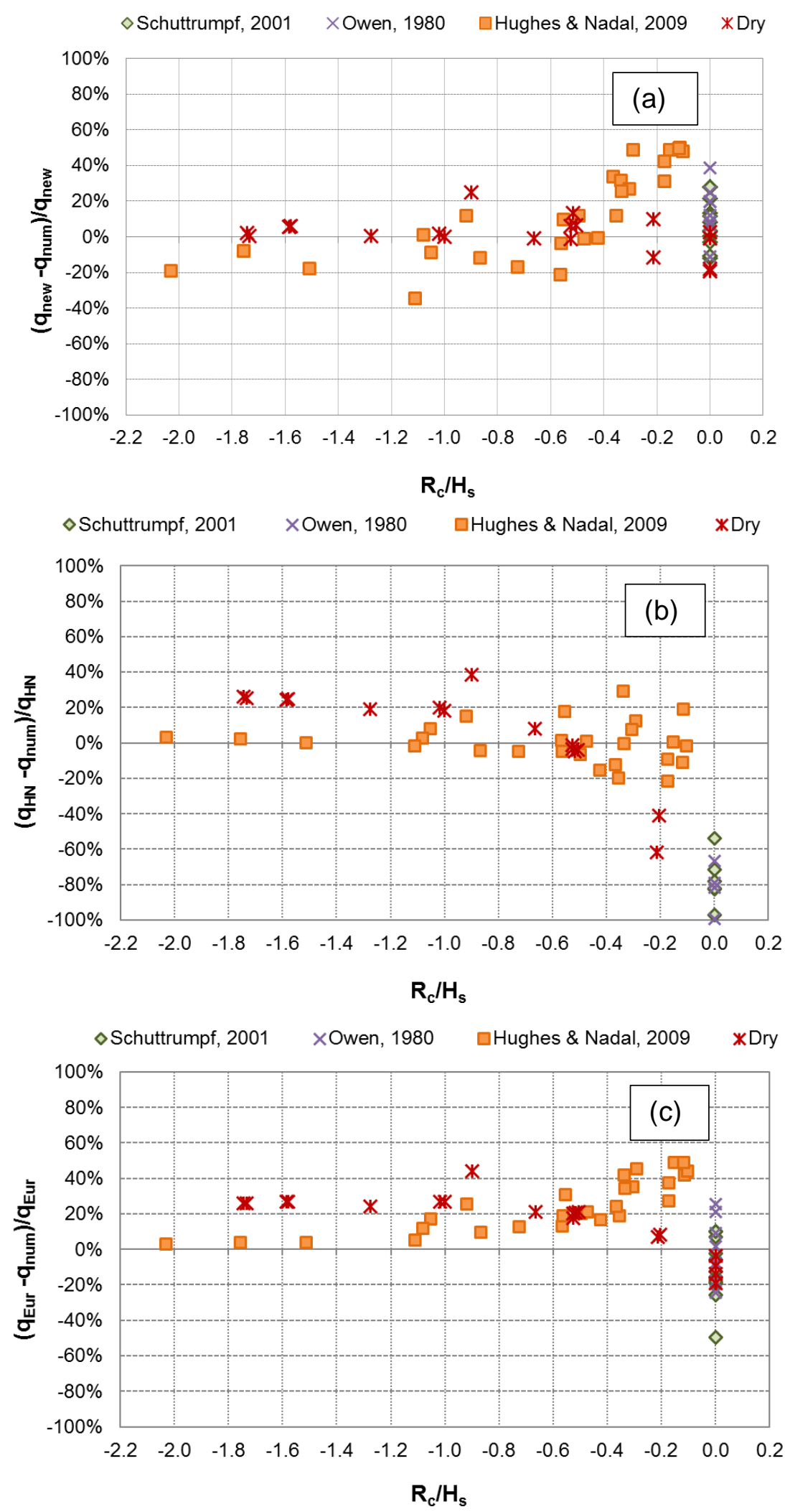

Figure 15 - Relative percentage errors among the values of $q$ derived from the numerical code $\left(q_{\text {num }}\right)$ and the corresponding estimations by: (a) the new fitting provided by Eq.s (6)-(7)-(8); (b) Hughes \& Nadal (2009), Eq.s (4) \& (5); (c) the EurOtop formulae, Eq.s (2)+(3). The errors are shown as functions of $R_{c} / H_{s}$. 
The best representation of the $\mathrm{HN}$ dataset is given by $q_{H N}$ (Figure $15, \mathrm{~b}$ ), while the tests at zerofreeboard are evidently out of the range of validity of the formula (i.e. $R_{d} / H_{S}<0$ ). $q_{H N}$ overestimates the dry tests of approximately the $20 \%$ when $R_{c} / H_{s} \leq-1$, gives a perfect representation around $R_{c} / H_{s}=-0.5$ and significantly under-predicts the numerical results when $R_{d} / H_{s}=-0.2$. Actually, this is expected since $q_{H N}$ was fitted on the $\mathrm{HN}$ dataset, which is characterized by significantly lower values of $q$ than the numerical results for similar submergence. This divergence among experimental and numerical values could be explained with the uncertainty associated to the reconstruction of the horizontal velocity values $(u)$ of the flow over the dike crest. For each time step $t, u(t)$ is the average of the punctual values of $u$ along the vertical in the numerical experiments, while in the lab experiments $u(t)$ is a punctual value measured at an elevation approximately half of the water depth of the steady overflow.

Overall, the following conclusions can be drawn.

- $q_{H N}$ provides the best representation of the laboratory measurements and a good estimation of the numerical dry tests, provided that $R_{c} / H_{s}<-0.3$. However, the method by Hughes \& Nadal (2009) cannot be applied neither to $R_{d} H_{s}=0$ nor to the wet tests.

- $q_{\text {Eur }}$ accurately reproduces the case of $R_{d} H_{s}=0$, with the exception of a couple of tests that are underestimated, and it generally gives cautious estimations of $q$ when $R_{d} / H_{s}<0$ for both the numerical dry tests and the laboratory measurements. Yet, the method is not targeted to represent the wet tests.

- $q_{\text {new }}$ is characterized by the best performance when applied to the numerical dry and wet tests, and provides a non-biased representation of most of the laboratory measurements for $R_{c} / H_{s} \leq 0$, revealing a degree of accuracy that is on average comparable to the existing methods.

Therefore, the new method represents a good compromise between accuracy and versatility, and it is the only method developed so far that is suitable to estimate $q$ in both dry and wet landward conditions for $R_{c} / H_{s} \leq 0$.

\subsection{Extension to overtopped and breached rubble mound breakwaters}

The accuracy of the new method in case of wet tests is here assessed by comparing the predictions $q_{\text {new }}$ with laboratory values of $q$ measured at rubble mound LCS in wet landward conditions. To this purpose, the following datasets have been selected:

- 9 tests conducted in the wave flume of the University of Firenze (Ruol et al., 2005); more precisely, the selected tests are characterized by $R_{c} / H_{s}=0, \cot \alpha_{\text {off }}=2, G_{c}=0.4 \mathrm{~m}$ (dataset $\mathrm{Fl}$-1, from now on).

- 30 tests performed by Cappietti et al. (2007) in the wave flume of the University of Firenze; these tests are characterized by $R_{c} / H_{s}=-0.05$ and 0 , cota $\alpha_{\text {off }}=2$ and $4, G_{c}=[0.2 ; 0.5] \mathrm{m}$ (dataset FI-2, from now on); 
- 4 tests carried out in the wave basin of the University of Bari by Martinelli et al. (2006), characterized by $R_{d} H_{s} \approx[-0.7 ;-0.3], G_{c}=0.33 \mathrm{~m}$ and $\cot \alpha_{\text {off }}=2$ (dataset BA, from now on).

- 20 tests by Kramer et al. (2005) in the shallow water wave basin of the University of Aalborg; the selected tests, characterized by $R_{d} / H_{s} \approx[-1.6 ; 0], G_{c}=0.2$ and $0.6 \mathrm{~m}$ and $\cot \alpha_{\text {off }}=2$ (dataset $A A U$, from now on).

The 3D tests include only non-oblique wave attacks. For these datasets, the new method has been applied by imposing the value of $\gamma_{f}=0.4$ in Eq. (8).

The performance of the new method against these datasets is reported in Table 6 in terms of $\sigma \%$ and $R^{2}$ and in Figure 16 in terms of comparison among predictions and measurements, respectively $q_{\text {new }}$ and $q_{\text {meas }}$.

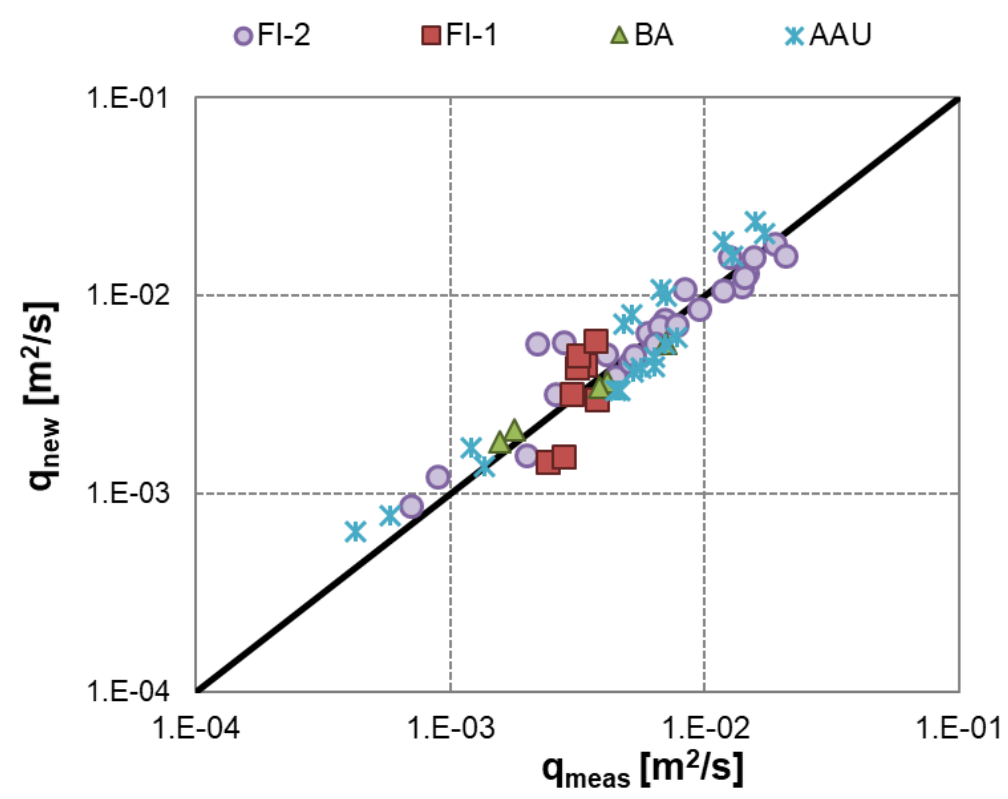

Figure 16 - Comparison among the values of $q$ computed with the new method $\left(q_{\text {new }}\right)$ and the corresponding measured values $\left(q_{\text {meas }}\right)$ for selected datasets on rubble mound LCS.

The performance indices of Table 6 indicate that the accuracy of the new method against LCS is comparable to its accuracy against over-washed dikes. In case of LCS indeed, the values of $\sigma \%$ are on average slightly greater, varying between $13 \%$ (BA and AAU) and $43 \%$ (FI-2), but the values of $R^{2}$ are closer to 1 , being the worst ( $\left.\mathrm{FI}-1\right)$ and the best (BA) cases $R^{2}=0.85$ and $R^{2}=1$, respectively. These values are also comparable to the performance of the existing methods $q_{\text {Eur }}$ and $q_{H N}$ applied to over-washed dikes (see Table 6). The slight tendency to overestimate $q_{\text {meas }}$, especially in submerged conditions, can be explained by the approximation of $R=0$.

In conclusion, the results of this application indicate that the new method can give reliable and accurate estimations of $q$ also in the case of rubble mound LCS in wet landward conditions. Such results are particularly satisfactory if considering that the method has been calibrated on smooth LCS exclusively. 


\section{Example application of the new method}

This section aims at providing the reader with the synthesis of the formulae included in the new method (Sub-section 6.1) and an application to a dike working in breached and over-washed conditions (Sub-section 6.2).

\subsection{The equations of the new method}

In summary, the method presented in this work consists of the following equations.

- For emerged structures $\left(R_{d} / H_{s}>0\right)$, both in dry and wet landward conditions, the wellconsolidated EurOtop formulae (Eq. 2) are recommended;

- In case of zero-freeboard and submerged structures $\left(R_{c} / H_{s} \leq 0\right)$, the new method synthesized in the Eq.s (6)-(7)-(8) is suggested, and specifically:

- for dry conditions, set $p_{u p}=0$ in Eq.(7) and in Eq. (10) and set the coefficients $a=0.055$ and $b=-0.35$ in Eq. (8);

- for wet conditions, compute $p_{u p}$ on the basis of Eq. (13) and set the coefficients $a=0.055$ and $b=1.2$ in Eq. (8).

For the practical use of the new method when $R_{c} / H_{s} \leq 0$, see the example application proposed in the next Sub-section 5.2.

\subsection{Example for a submerged structure}

The purposes of this Sub-section are i) to provide a simple and practical guide to apply the new method, and ii) to illustrate the resulting trend of $q$ against the main parameters $\left(R_{d} / H_{s}\right.$ and $H_{s} / L_{m-1,0}$ ) involved in the new formulae (Eq.s 6-7-8).

The geometrical features of the structure selected for the application and the wave conditions (input parameters, hereinafter) are resumed in Table 7. Starting from the original configuration, two different datasets have been artificially built up by varying the values of:

- the water depth $(h)$ and the structure freeboard $\left(R_{c}\right)$, while keeping constant the structure height $\left(h_{c}\right)$ and the significant incident wave height $\left(H_{s}\right)$, in order to evaluate the effects of the decreasing $R_{d} / H_{s}$;

- the spectral wave period $\left(T_{m-1,0}\right)$, for a constant $H_{s}$, to evaluate the sensitivity to $H_{s} / L_{m-1,0}$.

The values of the input parameters have been selected to test the applicability of the new methods beyond the range of the numerical simulations, i.e. beyond the range used to fit the new formulae. The input parameters required for the application of the new method are 7 (in green, in Table 7). The logical and computational steps to derive $q_{\text {overflow }}$ and $q_{\text {drift }}$ (and therefore q) are summarized in the scheme of Figure 17. The diagram starts (top green frame) with the list of the 7 input parameters and the values of the calibration coefficients necessary to compute all 
the "intermediate" quantities (middle blue frames) and ends with the final output quantities, $q_{\text {overflow }}$ and $q_{\text {drift }}$ (bottom orange frames). The 7 steps composing the diagram of Figure 17 can be easily implemented in a spreadsheet or organized in an algorithm (e.g. in the Matlab environment).

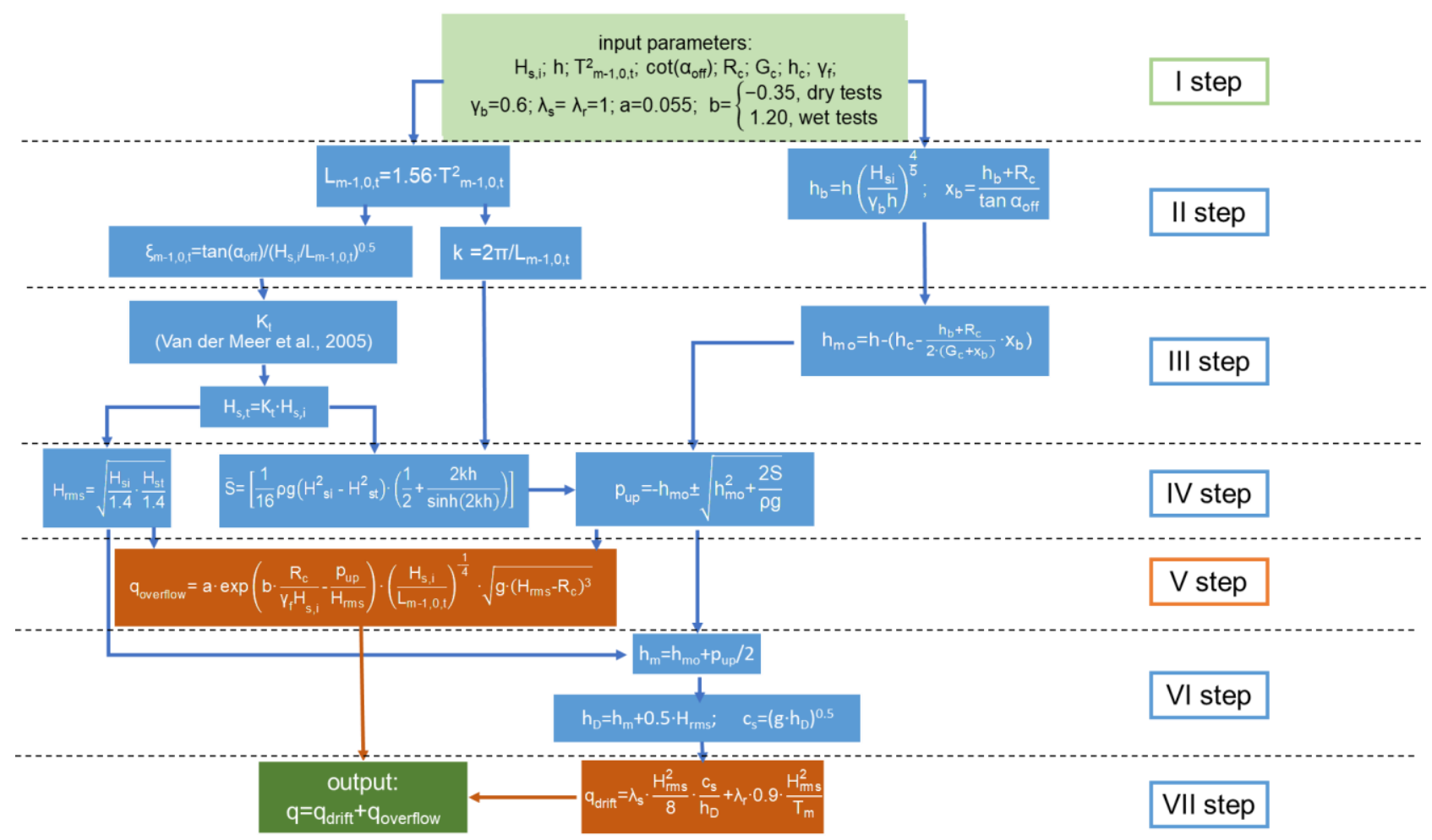

Figure 17 - Logical scheme resuming the steps required to apply the new formulae for the prediction of $q$.

Table 7. Hydraulic and geometrical parameters characterizing the example application of the new method. The reference for the symbols is given in Figure 1. To be continued.

\begin{tabular}{|c|c|c|c|}
\hline & \multicolumn{3}{|c|}{ Values } \\
\cline { 2 - 4 } & $\begin{array}{c}\text { Original } \\
\text { configuration }\end{array}$ & $\begin{array}{c}\text { Configuration with } \\
\text { varying } R_{c} / H_{s}\end{array}$ & $\begin{array}{c}\text { Configuration with } \\
\text { varying } H_{s} / L_{m-1,0}\end{array}$ \\
\hline $\begin{array}{c}\text { Input parameters } \\
\left(1^{\text {st }} \text { step}\right)\end{array}$ & & & 5 \\
\hline$h[\mathrm{~m}]$ & 5 & {$[5: 0.5: 8.8]$} & 2 \\
\hline$H_{s, i}[\mathrm{~m}]$ & 2 & 2 & $24: 0.5: 12]$ \\
\hline$T_{m-1,0, t}[\mathrm{~s}]$ & 6 & 6 & 0 \\
\hline $\cot \left(\alpha_{o f f}\right)$ & 2 & 2 & 4 \\
\hline$R_{c}[\mathrm{~m}]$ & 0 & {$[-3.8: 0.3: 0]$} & 5 \\
\hline$G_{c}[\mathrm{~m}]$ & 4 & 4 & 1 \\
\hline$h_{c}[\mathrm{~m}]$ & 5 & 5 & 1 \\
\hline$\gamma_{f}[-]$ & 1 & 1 & \\
\hline
\end{tabular}


Table 7. Continued.

\begin{tabular}{|c|c|c|c|}
\hline $\begin{array}{c}\text { Derived parameters } \\
\left(2^{\text {nd }} \text { step }\right)\end{array}$ & $\begin{array}{c}\text { Original } \\
\text { configuration }\end{array}$ & $\begin{array}{c}\text { Configuration with } \\
\text { varying } R_{d} / H_{s}\end{array}$ & $\begin{array}{l}\text { Configuration with } \\
\text { varying } H_{s} / L_{m-1,0, t}\end{array}$ \\
\hline$L_{m-1,0, t}=1.56 \cdot T^{2}{ }_{m-10, t}[\mathrm{~m}]$ & 56.2 & {$[25.0 ; 224.6]$} & \\
\hline$h_{b}[\mathrm{~m}]($ from Eq. 10) & 3.62 & [3.62;4.05] & 3.62 \\
\hline$x_{b}[\mathrm{~m}]$ (from Eq. 10) & 7.23 & {$[0.50 ; 7.23]$} & 7.23 \\
\hline$k=2 \pi / L_{m-1,0, t}$ & 0.112 & 0.112 & {$[0.028 ; 0.252]$} \\
\hline $\begin{array}{c}\xi_{m-1,0 t}= \\
=\tan \left(\alpha_{o f f}\right) /\left(H_{s, i} / L_{m-1,0, t}\right)^{0.5}\end{array}$ & 2.65 & {$[1.77 ; 5.30]$} & 2.65 \\
\hline \multicolumn{4}{|l|}{$\begin{array}{l}\text { Derived parameters } \\
\text { (3rd } s \text { step) }\end{array}$} \\
\hline $\begin{array}{c}K_{t}(\text { Van der Meer } \\
\text { et al., 2005) }\end{array}$ & 0.551 & {$[0.551 ; 0.800]$} & {$[0.440 ; 0.697]$} \\
\hline$H_{s, t}=K_{t} \cdot H_{s, i}[\mathrm{~m}]$ & 1.101 & {$[1.10 ; 1.60]$} & {$[0.880 ; 1.394]$} \\
\hline$h_{m o}[\mathrm{~m}]$ (from Eq. 9) & 1.16 & {$[1.16 ; 3.81]$} & 1.16 \\
\hline \multicolumn{4}{|l|}{$\begin{array}{l}\text { Derived parameters } \\
\qquad\left(4^{\text {th }} \text { step }\right)\end{array}$} \\
\hline$H_{\text {rms }}$ (from Eq. 8) & 1.060 & {$[1.060 ; 1.278]$} & [0.947;1.193] \\
\hline$S[\mathrm{~N} / \mathrm{m}]$ (from Eq. 11) & 2253 & [936;2253] & {$[1875 ; 2253]$} \\
\hline$p_{u p}[\mathrm{~m}]$ (from Eq. 12) & $\begin{array}{c}\text { wet: } 0.183 \\
\text { dry: } 0\end{array}$ & $\begin{array}{c}\text { wet: }[0.025 ; 0.183] \\
\text { dry: } 0\end{array}$ & $\begin{array}{c}\text { wet: }[0.148 ; 0.183] \\
\text { dry: } 0\end{array}$ \\
\hline \multicolumn{4}{|l|}{$\begin{array}{l}\text { Computation of qoverflow } \\
\left(5^{\text {th }} \text { step }\right)\end{array}$} \\
\hline$q_{\text {overflow }}\left[\mathrm{m}^{2} / \mathrm{s}\right]$ (from Eq. 7) & $\begin{array}{l}\text { wet: } 0.331 \\
\text { dry: } 0.433\end{array}$ & $\begin{array}{l}\text { wet: }[0.331 ; 0.644] \\
\text { dry: }[0.433 ; 8.823]\end{array}$ & $\begin{array}{l}\text { wet: }[0.232 ; 0.510] \\
\text { dry: }[0.299 ; 0.730]\end{array}$ \\
\hline \multicolumn{4}{|l|}{$\begin{array}{l}\text { Derived parameters } \\
\left.\text { ( } 6^{\text {th }} \text { step }\right)\end{array}$} \\
\hline$h_{m}[\mathrm{~m}]$ (from Eq. 9) & $\begin{array}{c}\text { wet: } 1.25 \\
\text { dry: } 1.16\left(=h_{m o}\right)\end{array}$ & $\begin{array}{c}\text { wet: }[1.24 ; 1.25] \\
\text { dry: }[1.16 ; 3.81]\left(=h_{m o}\right)\end{array}$ & $\begin{array}{l}\text { wet: }[1.25 ; 3.83] \\
\text { dry: } 1.16\left(=h_{m o}\right)\end{array}$ \\
\hline$h_{D}[\mathrm{~m}]\left(=h_{m}+0.5 \cdot H_{r m s}\right)$ & $\begin{array}{l}\text { wet: } 1.78 \\
\text { dry: } 1.69\end{array}$ & $\begin{array}{l}\text { wet: }[1.78 ; 4.46] \\
\text { dry: }[1.69 ; 4.45]\end{array}$ & $\begin{array}{l}\text { wet: }[1.71 ; 1.84] \\
\text { dry: }[1.64 ; 1.76]\end{array}$ \\
\hline$c_{s}[\mathrm{~m} / \mathrm{s}]\left(=\sqrt{g h_{D}}\right)$ & $\begin{array}{l}\text { wet: } 4.18 \\
\text { dry: } 4.08\end{array}$ & $\begin{array}{l}\text { wet: }[4.18 ; 6.62] \\
\text { dry: }[4.08 ; 6.61]\end{array}$ & $\begin{array}{l}\text { wet: }[4.10 ; 4.25] \\
\text { dry: }[4.01 ; 4.16]\end{array}$ \\
\hline \multicolumn{4}{|l|}{$\begin{array}{l}\text { Computation of } q_{\text {drift }} \\
\qquad\left(7^{\text {th }} \text { step }\right)\end{array}$} \\
\hline$q_{\text {drift }}\left[\mathrm{m}^{2} / \mathrm{s}\right]$ (from Eq. 6) & $\begin{array}{l}\text { wet: } 0.517 \\
\text { dry: } 0.525\end{array}$ & $\begin{array}{l}\text { wet: }[0.517 ; 0.635] \\
\text { dry: }[0.525 ; 0.648]\end{array}$ & $\begin{array}{l}\text { wet: }[0.493 ; 0.529] \\
\text { dry: }[0.499 ; 0.540]\end{array}$ \\
\hline \multicolumn{4}{|l|}{ Computation of $q$} \\
\hline$q\left[\mathrm{~m}^{2} / \mathrm{s}\right]$ (from Eq. 5) & $\begin{array}{l}\text { wet: } 0.848 \\
\text { dry: } 0.958\end{array}$ & $\begin{array}{l}\text { wet: }[0.848 ; 1.290] \\
\text { dry: }[0.958 ; 9.398]\end{array}$ & $\begin{array}{l}\text { wet: }[0.725 ; 1.113] \\
\text { dry: }[0.798 ; 1.269]\end{array}$ \\
\hline
\end{tabular}


The results of the application, i.e. the output values of $q$, are displayed in Figure 18 as functions of $R_{d} / H_{s}$ and $H_{s} / L_{m-1,0}$ (a and b, respectively). In each diagram, the predictions of $q$ computed with the new model are compared to the corresponding predictions given by the EurOtop formulae, Eq.s (2)+(3). The diagram with varying $R_{d} / H_{s}(\mathrm{a})$ includes also the comparison with the formulae by Hughes \& Nadal, Eq.s (4) and (5), which would provide a constant output against the varying $H_{s} / L_{m-1,0}$ (b).

The values of $q$ for $d r y$ and wet conditions are similar for the original configuration $\left(R_{c} / H_{s}=0\right.$ and $H_{s} / L_{m-1,0}=0.036$ ) and also when $H_{s} / L_{m-1,0}$ is made varying. A significant difference occurs instead with decreasing $R_{d} / H_{s}$ due to the qoverflow component (Table 7) that exponentially increases or is almost constant for dry or wet conditions respectively. The component $q_{\text {drift }}$ shows small changes for all the input configurations and for both the landward conditions.

As expected, Figure 18-a confirms that the new method gives values of $q$ very similar to the ones predicted by the existing methods when the dry condition is selected. In case of $R_{c} / H_{s}=0$ the method gives even more cautious estimates of $q$ than the EurOtop formulae, see Figure 17-b. The wet condition induces less cautious estimates and a significantly milder trend against $R_{c} / H_{s}$, that tends to become constant when $R_{d} / H_{s}>-1.5$.
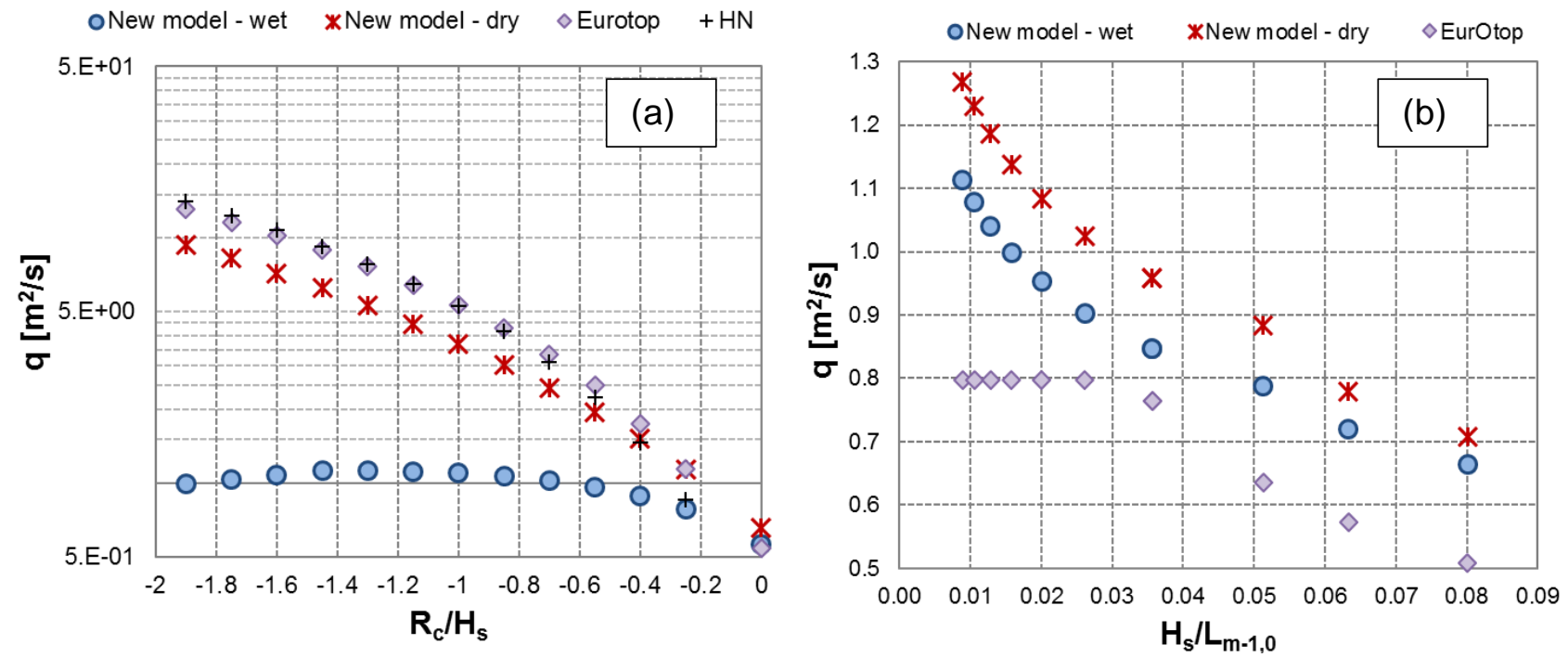

Figure 18 - Predicted values $q$ estimated with: the new model provided by Eq.s (6)-(7)-(8) for the dry and the wet tests; Eq.s (2)+(3); Eq.s (4) and (5). The predictions are shown as functions of $R_{\delta} / H_{s}(\mathrm{a})$ and of $H_{s} / L_{m-1,0}$ (b). 


\section{Conclusions}

This contribution presented a new conceptual and practical methodology for the prediction of the average wave overtopping discharge $(q)$ at dikes in climate change and catastrophic scenarios, i.e. in over-washed or completely breached conditions.

The new approach is founded on a coherent theoretical and physically-based framework, resulting into 3 practical formulae estimating the total overtopping rate $q$ as the sum of two separate contributions, i.e. the overflow ( $\left.q_{\text {overflow }}\right)$ and the drift $\left(q_{\text {drift }}\right)$ components. The formula for evaluating $q_{\text {drift }}$ is derived from the conceptual model already proposed in Zanuttigh et al. (2008). A new formulation has been conceived for qoverflow accounting for the combination of the 3 key factors governing the overflow process: the hydraulic head over the structure crest, determining the weir-like flow; the piling-up that may occur behind the structure and induce return flow over the crest; the wave breaking at the structure toe.

For the development and the calibration of the formulae, a new numerical database has been collected, consisting of 94 numerical simulations carried out against a smooth dike, characterized by a straight trapezoidal cross-section, with different off-shore slopes, variable crest levels (emerged, zero-freeboard and submerged) and subjected to a set of wave attacks. The simulations were performed with a slightly modified version of the original 2D-V RANS-VOF code (IH-2VOF model) developed by the University of Cantabria and validated in this contribution against experimental data.

The reliability of the numerical results was preliminary assessed by comparing the values of the wave reflection and transmission coefficients $\left(K_{r}\right.$ and $\left.K_{t}\right)$ to similar experimental data and to the predictions of $K_{r}$ and $K_{t}$ given by the ANN (Formentin et al., 2017). The comparison demonstrated that both the processes are accurately and consistently represented in the numerical code.

As expected, a good agreement among numerical measurements and theoretical predictions was found for all the emerged and over-washed dikes by comparing the numerical values of $q$ with the existing methods available from the literature, i.e. the formulae by EurOtop $(2007,2016)$ and by Hughes \& Nadal (2009). On the contrary, the submerged conditions related to breached dikes were substantially overestimated (up to one order of magnitude) by all the existing methods.

The accuracy and the reliability of the new method have been assessed based on the representation of the new numerical tests and of the existing laboratory data relative to smooth dikes in dry conditions (Owen, 1980; Schüttrumpf, 2001; Hughes \& Nadal, 2009) and rubble mound low-crested breakwaters in wet conditions (Cappietti et al., 2006; Clementi et al., 2006; Martinelli et al., 2006; Kramer et al., 2005). The best performance of the new method is achieved on the numerical database, where the values of the coefficient of determination $R^{2}$ computed among predictions and measurements are respectively equal to 0.99 and 0.96 for over-washed and breached conditions. 
For structures in dry conditions, the performance of the new method is not always higher than the existing formulae; however, differently from the other formulae, it provides always $R^{2}$-values greater than 0.80 . For breached structures, the new method tested against rubble mound LCS gives accurate results similarly to the dry conditions, with values of $R^{2}$ ranging between 0.85 and 1. Furthermore, the new method is the only one that can deal with both over-washed and breached structures achieving at least the same accuracy of the well-consolidated methods for overtopped dikes.

In conclusion, the new method presented in this work may represent so far the only alternative to numerical modelling for a quick and reasonable (i.e. non-overestimated) representation of the overtopping process in case of over-washed and breached dikes, scenarios that are likely to occur more often due to climate change.

\section{List of notations}

\begin{tabular}{|c|c|}
\hline a & Coefficient of the overflow component of the new method \\
\hline ANN & Acronym of Artificial Neural Network \\
\hline AAU & Contraction for the dataset by Kramer et al. (2005) \\
\hline$b$ & Coefficient of the overflow component of the new method \\
\hline BA & Contraction for the dataset by Martinelli et al. (2006) \\
\hline$C_{s}$ & Wave celerity over the structure crest, parameter of Eq. (7) \\
\hline$D_{50}$ & Nominal rock diameter or typical armour unit size \\
\hline$E$ & $\begin{array}{l}\text { Euclidean distance among the numerical tests and ANN field of validity, indicator } \\
\text { of the "similarity" between any new test (i.e. a test not used for the ANN training) } \\
\text { test and the tests used to train the ANN }\end{array}$ \\
\hline $\mathrm{FI}-1$ & Contraction for the dataset by Ruol et al. (2005) \\
\hline $\mathrm{FI}-2$ & Contraction for the dataset by Cappietti et al. (2007) \\
\hline$g$ & Acceleration due to gravity \\
\hline$G_{c}$ & Structure crest width \\
\hline$h$ & Still water depth (in generation) or flow depth (over the structure crest) \\
\hline$H$ & Wave height (monochromatic waves) \\
\hline$h_{b}$ & Breaker depth \\
\hline$h_{c}$ & Structure crest height \\
\hline$h_{D}$ & $\begin{array}{l}\text { Average water depth over the structure crest accounting of the wave pumping, } \\
\text { parameter of Eq. ( } 7 \text { ) }\end{array}$ \\
\hline$h_{m}$ & $\begin{array}{l}\text { Average water depth over the crest between the wave breaking point and the } \\
\text { breaking end, used to compute } h_{D}\end{array}$ \\
\hline$h_{m o}$ & $\begin{array}{l}\text { Water depth over the crest between the wave breaking point and the breaking } \\
\text { end, used to compute } h_{m} \text { or coincident to } h_{m} \text { if } p_{u p}=0 \text { in Eq. (10) }\end{array}$ \\
\hline
\end{tabular}




\begin{tabular}{|c|c|}
\hline$H_{r m s}$ & $\begin{array}{l}\text { rms wave height computed as the geometric mean between the rms transmitted } \\
\left(H_{r m s, t}\right) \text { and the rms incident }\left(H_{r m s, i}\right) \text { wave heights, parameter of Eq. }(7)\end{array}$ \\
\hline$H_{r m s, i}$ & rms incident wave height, used to compute $H_{r m s}$ in Eq. (9) \\
\hline$H_{r m s, t}$ & rms transmitted wave height, used to compute $H_{r m s}$ in Eq. (9) \\
\hline$H_{s}$ & Simplified notation of $H_{s i}$ (significant incident wave height) \\
\hline$H_{s i}$ & Significant incident wave height \\
\hline$H_{s t}$ & Significant transmitted wave height \\
\hline $\mathrm{HN}$ & Contraction of "Hughes \& Nadal dataset, 2009" \\
\hline$k$ & Wave number, component of $\bar{S}$, Eq. (12b) \\
\hline$K_{r}$ & Wave reflection coefficient \\
\hline$K_{r, Z V D M}$ & Wave reflection coefficient predicted by Eq. (1) \\
\hline$K_{r, \text { num }}$ & Wave reflection coefficient derived from the numerical simulations \\
\hline$K_{t}$ & Wave transmission coefficient \\
\hline$m$ & Coefficient depending on $\gamma_{f}$ in Eq. (1) \\
\hline$n$ & Coefficient depending on $\gamma_{f}$ in Eq. (1) \\
\hline$L$ & Wave length (monochromatic waves) \\
\hline$L_{m-1,0, t}$ & Wave length based on spectral wave period at the structure toe \\
\hline LCS & Acronym of low-crested structures \\
\hline$p_{\text {up }}$ & Piling-up (respect to the still water level) measured behind the structures \\
\hline OW & Contraction of "Owen dataset, 1980" \\
\hline$q$ & Average specific wave overtopping discharge \\
\hline$q_{A N N}$ & Average wave overtopping discharge predicted by the ANN \\
\hline$q_{\text {drift }}$ & Drift component of the average wave overtopping discharge \\
\hline$q_{\text {Eur }}$ & Average wave overtopping discharge predicted by Eq.s (2) + (3) \\
\hline$q_{H N}$ & Average wave overtopping discharge predicted by Eq.s (4) \& (5) \\
\hline$q_{\text {meas }}$ & Average wave overtopping discharge derived from laboratory measurements \\
\hline$q_{\text {new }}$ & $\begin{array}{l}\text { Average wave overtopping discharge predicted by the new method, } \\
\text { Eq.s }(6),(7),(8)\end{array}$ \\
\hline$q_{\text {num }}$ & Average wave overtopping discharge derived from the numerical simulations \\
\hline qoverflow & Overflow component of the average wave overtopping discharge \\
\hline qovertop & Overtopping component of the average wave overtopping discharge \\
\hline$u$ & Horizontal component of the flow velocity in the numerical simulations \\
\hline $\bar{R}$ & $\begin{array}{l}\text { Resultant of the friction force over the crest dike, component of the cross-shore } \\
\text { momentum equation, Eq. (12) }\end{array}$ \\
\hline$R^{2}$ & Coefficient of determination \\
\hline$R_{c}$ & $\begin{array}{l}\text { Structure freeborard (negative if the structure is submerged) with the respect to } \\
\text { the still water level }\end{array}$ \\
\hline$r m s$ & Acronym of "root mean squared" \\
\hline RANS & Acronym of "Reynolds-Averaged Navier Stokes equations" \\
\hline
\end{tabular}




\begin{tabular}{|c|c|}
\hline $\bar{S}$ & $\begin{array}{l}\text { Radiation stress or resultant momentum excess due to the waves, component of } \\
\text { the cross-shore momentum equation, Eq. (12) }\end{array}$ \\
\hline$S_{0}$ & Wave steepness based on spectral wave period at the structure toe \\
\hline SC & Contraction of "Schüttrumpf dataset, 2001" \\
\hline$T$ & Wave period (monochromatic waves) \\
\hline$T_{m-1,0}$ & Spectral wave period \\
\hline$T_{m}$ & Mean period from spectral analysis at the structure toe $=\mathrm{m}_{2} / \mathrm{m}_{0}\left(\right.$ see $\left.\mathrm{m}_{\mathrm{n}}\right)$ \\
\hline$T_{p}$ & Peak period from spectral analysis \\
\hline VOF & Acronym of "Volume Of Fluid" \\
\hline$x$ & Label representing the horizontal direction in the numerical domain \\
\hline$x_{b}$ & $\begin{array}{l}\text { Distance between the breaking point and the seaward crest edge, to be computed } \\
\text { from Eq. (11) and used in Eq. (10) }\end{array}$ \\
\hline$z$ & Label representing the vertical direction in the numerical domain \\
\hline$\alpha_{i n}$ & On-shore slope of a structure \\
\hline$\alpha_{\text {off }}$ & Off-shore slope of a structure \\
\hline$\gamma_{b}$ & Breaker index, according to Battjes and Janssen (1978) \\
\hline$\gamma_{f}$ & Roughness factor as found in overtopping research \\
\hline$\Delta \mathrm{x}$ & Horizontal size of the computational mesh \\
\hline$\Delta \mathrm{z}$ & Vertical size of the computational mesh \\
\hline$\varepsilon$ & Turbulent dissipation rate in the RANS-VOF numerical code \\
\hline$K$ & Turbulent kinetic energy in the RANS-VOF numerical code \\
\hline$\lambda_{r}$ & Calibration coefficient of the $q_{\text {drift }}$ representing the roller mass drift, Eq. (7) \\
\hline$\lambda_{s}$ & Calibration coefficient of the $q_{\text {drift }}$ representing the Stokes drift, Eq. (7) \\
\hline$\xi_{0, m-1}$ & Iribarren-Battjes breaker parameter based on spectral wave period \\
\hline $\bar{\Pi}$ & $\begin{array}{l}\text { Resultant pressure force on the control volume surface, component of the cross- } \\
\text { shore momentum equation, Eq. (12) }\end{array}$ \\
\hline$\sigma$ & Standard deviation \\
\hline$\sigma \%$ & Normalized percentage standard deviation \\
\hline
\end{tabular}

\section{Acknowledgments}

The authors would like to express their sincere gratitude to Professor S. A. Hughes for providing his own experimental data on wave overtopping and overflow we used for the validation of the numerical model.

The support of the European Commission through Contract 244104 THESEUS ("Innovative technologies for safer European coasts in a changing climate"), FP7.2009-1 Large Integrated Project, is gratefully acknowledged for funding the whole $\mathrm{PhD}$ of the first author and this activity specifically. 


\section{References}

Battjes, J.A. and Janssen, J.P.F.M. Energy loss and set-up due to breaking of random waves. Proc. $16^{\text {th }}$ International Conference of Coastal Engineering, New York, USA, Vol. 16, ASCE, 1978, pp. 569-587.

Calabrese, M., Vicinanza, D. and Buccino M. 2D wave set up behind low crested and submerged breakwaters. Proc. $13^{\text {th }}$ International Conference ISOPE, Honolulu, Hawaii, USA, 2003, pp.831836.

Calabrese, M., Vicinanza, D. and Buccino M. Verification and recalibration of an engineering method for predicting 2D wave setup behind submerged breakwaters. Proc. International Coastal Symposium, Hofn, Iceland, 2005.

Cappietti L., Clementi E., Aminti P. and Lamberti, A. Piling-up and filtration at low crested breakwaters of different permeability. Coastal Engineering, 2006, pp. 4957-4969.

Cox, R.J and Tajziehchi, M. 2D experimental modelling of hydrodynamic effects of submerged breakwaters. Proceedings of the 5th International Conference on Coastal Dynamics, Barcelona, Spain, 2005.

EurOtop. European Manual for the Assessment of Wave Overtopping. T. Pullen, N.W.H. Allsop, T. Bruce, A. Kortenhaus, H. Schüttrumpf and J.W. van der Meer, 2007. At: www.overtoppingmanual.com.

EurOtop. Manual on wave overtopping of sea defences and related Structures. An overtopping manual largely based on European research, but for worldwide application. Second Edition.N.W.H. Allsop, T. Bruce, J. DeRouck, A. Kortenhaus, T. Pullen, H. Schüttrumpf, P. Troch, J.W. van der Meer and B. Zanuttigh, 2016. www.overtopping-manual.com

Formentin S.M., Zanuttigh B. and J.W. van der Meer. A neural network for predicting wave reflection, overtopping and transmission, Coastal Engineering Journal, 59, No. 2, 1750006, 2017, 31 pp.

Garcia, N., Lara, J.L., Losada, I.J. 2-D numerical analysis of near-field flow at low-crested breakwaters. Coastal Engineering 51 (10), 2004, pp.991-1020.

Hsu, T.-J. A two-phase flow approach for sediment transport. PhD thesis, University, Ithaca, NY, USA, 2002.

Hughes, S. A., and Nadal, N. C. Laboratory study of combined wave overtopping and storm surge overflow of a levee, Coastal Engineering, Elsevier, 56(3), 2009, pp.244-259.

Hughes, S.A., and Shaw, J.M. Continuity of instantaneous wave overtopping discharge with application to stream power concepts, Journal of Waterway, Port, Coastal and Ocean Engineering, ASCE, 137(1), 2011, pp.12-25. 
Hughes, S.A. Thornton, J.W. van der Meer and B. Scholl. Improvements in describing wave overtopping processes. ASCE, Proc. ICCE, 2012, Santander, Spain.

Johnson H. K., Karambas T., Avgeris I., Zanuttigh B., Gonzalez-Marco D., Caceres. Modelling of waves and currents around submerged breakwaters, Coastal Engineering, 52, 2005, pp.949969

Kothe D.B., Mjolsness R. C., M. D.J. Torrey. RIPPLE: A computer program for incompressible flows with free surfaces, Report LA-12007, Los Alamos National Laboratory, 1991.

Kramer, M., Zanuttigh, B., van der Meer J. W., Vidal, C. and Gironella, X. 2D and 3D experiments on low-crested structures, Coastal Engineering, 52, 2005, pp.867-88.

Lara, J.L., Losada, I.J., Liu, P.L.-F. Breaking waves over a mild gravel slope: experimental and numerical analysis. Journal of Geophysical Research, AGU, Vol. 111, C11019, 2006. doi: 101029/2005 JC003374.

Lara J.L., Losada I.J., R. Guanche. Wave interaction with low-mound breakwater using a rans model. Ocean Engineering, 56, 2008, pp.543-558.

Lara, J.L., Ruju, A. and Losada, I.J. Reynolds Averaged Navier-Stokes modelling of long waves induced by a transient wave group on a beach. Proceedings of the Royal Society A, vol. 467, 2011, pp.1215-1242.

Lin P., Liu, P.L.-F. A numerical study of breaking waves in the surf zone. Journal of Fluid Mechanics, 359, 1998, pp.239-264.

Liu, P.L.-F., Lin, P. A numerical model for breaking waves: the volume of fluid method. Research Report No. CACR-97-02, Center for Applied Coastal Research, Ocean Engineering Laboratory, University of Delaware, Newark, DE, 1997.

Liu, P.L.-F., Lin, P., Chang, K.-A., Sakakiyama, T. Numerical modelling of wave interaction with porous structures. J. Waterw., Port, Coast., and Ocean Eng., 125 (6), 1999, pp.322 - 330.

Losada, I.J., Lara J.L., Christensen, E.D. and Garcia N. Modelling of velocity and turbulence fields around and within low-crested rubble-mound breakwaters, Coastal Engineering, 52(1011), 2005, pp.887-913.

Losada, I.J., J.L. Lara, R. Guanche and J.M. Gonzalez-Ondina. Numerical analysis of wave overtopping of rubble mound breakwaters, Coastal Engineering, 55(1), 2008, pp. 47-62.

Martinelli, L., Zanuttigh, B. and Lamberti, A. Hydrodynamic and morphodynamic response of isolated and multiple low crested structures: Experiments and simulations, Coastal Engineering 53(4), 2006, pp. 363-379. 
Misra, S., Narayanaswamy, M., Bayram, A., and Shi, F., 2011. Optimization of caisson breakwater superstructure geometry using a 2DV Rans-Vof numerical model. Coastal Engineering Proceedings, 1(32).

Muttray, M., Oumeraci, H. and Ten Oever, E. Wave Reflection and Wave Run-Up at Rubble Mound Breakwaters. Proc. $30^{\text {th }}$ International Conference of Coastal Engineering, San Diego, USA, 2006.

Owen M.W., Design of seawalls allowing for wave overtopping, Hydraulic Research Institute Wallingford, Report EX924, June 1980.

Peng Z., Zou Q.P. Spatial distribution of wave overtopping water behind coastal structures, Coastal Engineering 58(6), 2011, pp.489-498.

Reeve, D.E., Soliman, A. and Lin P.Z. Numerical study of combined overflow and wave overtopping over a smooth impermeable seawall, Coastal Engineering 55(2),2008, pp.155-166.

Rodi, W. Turbulence models and their application in hydraulics - a state-of-the-art review. IAHR Publication, 1980, 104 pp.

Ruol P., Martinelli L., Zanuttigh B.; Cappietti L.; Vicinanza D.; Faedo P. Sui fenomeni di tracimazione, filtrazione e piling-up per opere di difesa longitudinale a cresta bassa, Studi costieri, 2005, 9 pp (in italian).

Schüttrumpf, H.F.R. Wellenüberlaufströmung bei See-deichen, Ph.D.-thesis, Technical University Braunschweig, 2001.

Schüttrumpf H., H. Oumeraci. Layer thicknesses and velocities of wave overtopping flow at sea dikes, Coastal Engineering, 52 (6), 2005, pp.473-495.

Seed, R.B., Bea, R.G., Abdelmalak, R.I., Athanasopoulos-Zekkos, A., Boutwell, G.P., Briaud, J.L., Cheung, C., Cobos-Roa, D., Ehrensing L., Govindasamy, A.V., Harder L.F., Inkabi K.S., Nicks, J., Pestana, J.M., Porter, J., Rhee, K., Riemer, M.F., Rogers, J.D., Storesund, R., VeraGrunauer, X. and Wartman, J. New Orleans and Hurricane Katrina. I: Introduction, Overview, and the East Flank, Journal of Geotechnical and Geoenvironmental Engineering, 134(5), 2008, ASCE.

Tajziehchi, M. Experimental and numerical modelling of wave-induced current and wave transformation in presence of submerged breakwaters, Ph.D.-thesis, Civil \& Environmental Engineering, Faculty of Engineering, UNSW, 2006.

Torres-Freyermuth, A., Lara, J.L. and Losada, I.J. Numerical modelling of short and long-wave transformation on a barred beach. Coastal Engineering, vol. 57, 2010, pp.317-330.

Van der Meer, J.W., Briganti, R., Zanuttigh, B. and B. Wang. Wave transmission and reflection at low crested structures: design formulae, oblique wave attack and spectral change, Coastal Engineering, 52 (10-11), 2005, pp.915-929. 
Van der Meer, J.W., R. Schrijver, B. Hardeman, A. van Hoven, H. Verheij and G.J. Steendam (a). Guidance on erosion resistance of inner slopes of dikes from three years of testing with the Wave Overtopping Simulator. Proc. ICE, Breakwaters, Marine Structures and Coastlines; Edinburgh, UK, 2009.

Van der Meer, J.W., Verhaeghe, H. and Steendam, G.J (b). The new wave overtopping database for coastal structures. Coastal Engineering 56, 2009, pp.108-120.

Van Gent, M.R.A., van den Boogaard, H.F.P., Pozueta, B. and Medina, J.R. Neural network modelling of wave overtopping at coastal structures. Coastal Engineering 54, 2007, pp.586-593.

Zanuttigh, B. and J.W. van der Meer. Wave reflection from coastal structures in design conditions, Coastal Engineering, 2008.

Zanuttigh, B., Martinelli L. and Lamberti A. Wave overtopping and piling-up at permeable low crested structures, Coastal Engineering 55, 2008, pp.484-498.

Zanuttigh B., Formentin S.M., and Van der Meer J.W. Prediction of extreme and tolerable wave overtopping discharges through an advanced neural network, Ocean Engineering, 127, 2016, pp.7-22.

Zelt, J.A., Skjelbreia, J.E. Estimating incident and reflected wave field using an arbitrary number of wave gauges. Proc. 23 ${ }^{\text {rd }}$ International Conference of Coastal Engineering 1992, vol I, pp. 777789. 